\title{
MERCADOS DE TRABAJO EN LA CIENCIA Balance de la investigación y propuesta de marco analítico
}

\author{
MANUEL FERNÁNDEZ ESQUINAS \\ IESA-CSIC. Córdoba
}

\author{
PALABRAS CLAVE ADICIONALES \\ ADDITIONAL KEYWORDS
Science, LabourMarket, Occupations, Operative \\ Ciencia, Mercado de trabajo, Ocupaciones, \\ Operacionalización. \\ Definition.
}

RESUMEN. El mercado de trabajo en las instituciones científicas, además de ser un proceso de compra-venta de fuerza de trabajo, es el ámbito en el que se produce la intersección entre los principios de funcionamiento de la ciencia contemporánea y los procedimientos de trabajo de los grupos y las organizaciones científicas, entre las capacidades y actitudes de los trabajadores y los criterios empleados para adaptarlos a los procesos del trabajo de investigación. Por ello, el mercado de trabajo constituye un elemento privilegiado para observar el funcionamiento de la investigación científica en sus diversos marcos organizativos y es un factor clave en el desarrollo de cualquier sistema de I+D. En este trabajo se realiza una revisión de las principales aportaciones que han tratado el proceso de incorporación y movilidad de trabajadores científicos y la estructuración del sistema de ocupaciones en la ciencia, se expone un marco analítico que utiliza los conocimientos acumulados en este área de problemas y se desarrolla un esquema operativo para la investigación empírica de los diversos mercados de trabajo tanto en la ciencia académica como en la industrial.

SUMMARY. The job market in scientific institutions, in addition to buying and selling the workforce, is the crossroads where the operational principles of contemporary science and the working procedures of scientific groups and organizations come together. It is in this intersection where the skills and attitudes of scientific workers and the criteria employed to adapt them to research processes meet face to face. A key factor in the development of any R\&D system, the job market is an ideal setting to observe how scientific research functions within its different organizational frameworks. This study provides an overview of the most important contributions concerning the incorporation and mobility of workers and the occupational structure in the sciences. An analytical framework and operating scheme are proposed for the empirical investigation of job markets in both academic and industrial science.

E-mail: mfernandez@iesaa.csic.es

Revista Internacional de Sociología (RIS)

Tercera Época, nº 32, Mayo-Agosto, 2002, pp. 35-75. 
RIS

REVISTA INTERNACIONAL DE SOCIOLOGÍA

NN 32, Mayo-Agosto, 2002

MANUEL FERNÁNDEZ ESQUINAS

\section{INTRODUCCIÓN}

Si bien las ciencias sociales siempre han estado dispuestas a estudiar cualquier tipo de institución humana, no lo han estado tanto cuando han tenido que estudiar la institución científica a la que ellas mismas pertenecen. Aunque los Estudios Sociales de la Ciencia tienen ya una tradición de más de medio siglo, algunos de sus aspectos fundamentales distan de haberse constituido como problema de investigación que genere estudios empíricos en los diversos ámbitos organizativos y culturales de una forma continuada. Este es el caso del mercado de trabajo científico, cuestión que, con notables excepciones, se ha considerado como uno de los puntos de observación del modo de funcionamiento de la ciencia dentro de los enfoques que se dirigían a indagar las características de la ciencia como institución y, en última instancia, a definir su naturaleza desde un puntó de vista más amplio. De este modo, los estudios sobre este tema han ocupado un lugar subsidiario, bien como subproblema de investigación, bien como subproducto de dichos estudios.

Sin restar importancia a los enfoques existentes tanto en la economía como en la sociología de la ciencia, en este artículo se sostiene que el mercado de trabajo es uno de los problemas de estudio fundamentales en la institución científica contemporánea. Primero, porque constituye un problema que en sí mismo presenta interés por la importancia que los recursos humanos adquieren en cualquier sistema de $\mathrm{I}+\mathrm{D}$, debido a que son el principal recurso de la investigación científica. Segundo, porque el mercado de trabajo es una de las vías privilegiadas para observar el funcionamiento real de las instituciones y de los grupos de científicos al ser uno de los puntos claves de la estructuración social de la ciencia. De este modo, es necesario contar con un marco analítico adecuado que de cuenta de los procesos sociales que subyacen al funcionamiento del mercado de trabajo. El objetivo principal de este trabajo es establecer un marco de análisis que pueda utilizarse en términos operativos en la investigación de los mercados de trabajo científico partiendo de dos supuestos: el conocimiento acumulado en los Estudios Sociales de la Ciencia y sus posibilidades para la investigación empírica en los asuntos que se consideran más relevantes en los principales entornos de la ciencia contemporánea, a saber, la ciencia académica, la ciencia de los institutos oficiales de investigación y la ciencia aplicada de las corporaciones industriales.

\section{PERSPECTIVAS DE ANÁLISIS EN EL ESTUDIO DE LOS MERCADOS DE TRABAJO EN LA CIENCIA}

La investigación del mercado de trabajo en la ciencia procede de dos grupos de tradiciones disciplinarias, la económica y la sociológica, ambas con varios tipos de enfoques, unas veces complementarios, otras veces excluyentes. A pesar de 
que el contenido de este trabajo se encuadra en el ámbito de la sociología, se considera conveniente partir de los enfoques económicos debido a que definen áreas de problemas relevantes y ayudan a perfilar mejor los enfoques sociológicos, para centrarnos posteriormente en las diversas perspectivas que se encuadran en algunas de las tradiciones del pensamiento sociológico en torno a la ciencia. Sin ánimo de realizar una exposición exahustiva de todos los estudios que han tratado central o tangencialmente el asunto del mercado de trabajo, aquí se han seleccionado los grupos de investigaciones considerados más importantes por sus aportaciones teóricas o empíricas con una intención más integradora que crítica. Debido a la complejidad que plantea el estudio de la ciencia como institución, se considera necesario plantear como objetivo un enfoque comprehensivo que tenga en cuenta los hallazgos más relevantes que se han producido para obtener un adecuado entendimiento del proceso social en que opera la incorporación, movilidad y salida de trabajadores en el mundo científico ${ }^{1}$.

\section{Las perspectivas económicas}

Uno de los elementos fundamentales de los enfoques económicos es la consideración del mercado de trabajo científico, al igual que el mercado de trabajo en general, en términos de oferta y demanda, del proceso de compra-venta de trabajo en el ámbito de lo que se considera ciencia, de los factores influyentes y de los resultados de dicho proceso en térmicos productivos ${ }^{2}$. La ciencia suele ser considerada como un mercado diferenciado por las características de su fuerza de trabajo en términos de cualificación y por las particularidades de su estructura ocupacional. De este modo, los elementos propios de estos enfoques se centran en los procesos que inciden en la creación de puestos de trabajo y la ocupación de los mismos -la demanda-y la disposición de los trabajadores para integrarse en el mundo laboral -la oferta. En la exposición de algunos estudios relevantes del mercado de trabajo en la ciencia se toma como punto de partida uno de los 'núcleos fuertes' de la teoría económica, el enfoque del capital humano, para continuar con enfoques que incorporan otros elementos como las perspectivas de la selección y las perspectivas institucionales ${ }^{3}$.

\footnotetext{
${ }^{1}$ Aquí se parte de una definición amplia del mercado de trabajo, la que lo considera como el ámbito en el que los trabajadores intercambian su fuerza de trabajo por salarios, estatus u otras recompensas laborales (Kallenberg y Sorensen,1979).

${ }^{2}$ La definición de la ciencia que suele subyacer a estas perspectivas no es la que considera a la ciencia como institución social, sino como conjunto de puestos y trabajadores en el sector de la ciencia.

${ }^{3}$ Aunque a algunas de las ideas que aquí se exponen se les atribuye el estatus de 'teorías', aquí se prefiere emplear el término 'enfoque' o 'marco analítico' debido a que, en lo referente al mercado
} 
RIS

REVISTA INTERNACIONAL DE SOCIOLOCIA

No 32, Mayo-Agosto, 2002

\section{El enfoque del capital humano}

El enfoque del capital humano surge en la estela de la economía neoclásica, que ya venía modificando el papel asignado al trabajo en los análisis económicos, en contraste con las ideas ortodoxas que habían tratado el trabajo como un tipo de mercancía. A saber, las que suponían que la oferta de trabajo estaba determinada por el stock de población en situación de trabajar, mientras que la demanda era vista como uno más de los factores de producción de que disponían los empleadores. Así, era una demanda derivada de la demanda de producto y de la técnica concreta de producción utilizada, lo que, en principio, no presentaba notables diferencias con otros factores productivos ${ }^{4}$.

Las teorías económicas neoclásicas modifican dicho enfoque tomando en consideración la complejidad del trabajo en la creación de riqueza e introduciendo como elemento fundamental las características de la mano de obra de los países desarrollados. En este caso, serían los niveles de rendimiento de los distintos grupos de fuerza de trabajo los que se traducen en un mayor nivel de productividad y renta en los países con mayores inversiones en capital humano. Aunque este enfoque adopta el planteamiento neoclásico supone un salto cualitativo al considerar el trabajo no sólo como mercancía, sino como capital. La idea fundamental es que las personas gastan en sí mismas, no para obtener satisfacciones inmediatas, sino para obtener ingresos monetarios y no monetarios en el futuro, siendo este gasto visto más como inversión que como consumo de acuerdo con una perspectiva utilitaria del comportamiento. Dicho principio ha sido aplicado profusamente a numerosos comportamientos individuales tales como la inmigración, el matrimonio o la familia, pero el campo de mayor aplicación ha sido la educación. Desde este punto de vista, la educación es una inversión en la vida de las personas que revaloriza el trabajo posterior en función de la cantidad y calidad de educación aportada. Las personas demandarán más o menos educación en función de los costes privados directos o indirectos de la enseñanza y de las expectativas de ingresos asociados a los años de enseñanza. Por tanto, la elección de educación es el resultado de poner en la balanza personal de cada individuo la diferencia entre ingresos presentes y futuros. La inversión en capital humano

\footnotetext{
científico, no suelen estár formalizadas como tales y las investigaciones que generan tampoco suelen contrastar todas sus proposiciones. No obstante, a efectos expositivos se emplean las palabras enfoque y teoría indistintamente.

${ }^{4} \mathrm{Si}$ bien el empleo de los elementos tradicionales constituidos por el capital y el trabajo no explicaba suficientemente el crecimiento económico en numerosos países, la parte no explicada era tratada frecuentemente como 'residuo'. Sobre los supuestos generales de este enfoque ver, por ejemplo, Mincer (1971).
} 
es vista, pues, como el sacrificio de una renta actual a cambio de un beneficio mayor en el futuro 5 .

La formalización más comúnmente aceptada de estos presupuestos se encuentra en trabajo de Gary Becker dedicado a la explicación de los determinantes de los ingresos procedentes del empleo (Becker, 1983), donde se expone cómo la capacidad y la escolarización se traducen en retornos al mercado de trabajo, y cómo los atributos y los conocimientos de un individuo generan logros ocupacionales y económicos en diferentes puntos del curso de su vida ${ }^{6}$. La inversión en capital humano contenida en una persona se convierte, pues, en uno de los determinantes de la calidad del trabajo para el que está capacitado. Este hecho será decisivo en la elección del empleador, ya que conlleva un determinado 'nivel de calidad' como factor de producción. Y también será decisivo en la generación de riqueza debido a que el proceso económico descrito provoca que confluyan en el mercado cualificaciones profesionales y demandas de los empleadores, lo cual eleva los niveles de productividad en cualquier parcela económica.

En lo referido al trabajo científico, existen tempranos estudios sobre los profesionales académicos que examinaban los efectos de la educación superior en los ingresos, demostrando que los retornos a la educación graduada son generalmente importantes, aun habiendo diferencias entre distintos sectores laborales (Stigler y Blanc, 1957; Cartter, 1965). Más recientemente, los análisis más extensos de los mercados de trabajo en la ciencia son los realizados por Richard Freeman, en los que se lleva a cabo una comprobación de cómo los jóvenes realizan inversiones racionales en capital humano y de qué factores contribuyen a explicar los determinantes de la oferta de trabajo científico. En concreto, Freeman buscaba en sus investigaciones evidencias empíricas de la sensibilidad de la oferta a los incentivos económicos en aquellos grupos en los que resultara más probable este comportamiento (Freeman, 1971; 1976; 1994). Es decir, se buscaba la influencia del incentivo salarial en la decisión de los jóvenes en invertir en educación y, por tanto, en la oferta de titulados universitarios. Y los grupos donde podía observar dicho comportamiento los encontró en aquellas personas que realizaban inver-

\footnotetext{
${ }^{5} \mathrm{~A}$ partir de estas premisas, el programa del capital humano se ha encaminado a explicar dos cuestiones básicas. En primer lugar, la búsqueda de los retornos educativos en forma de salarios a lo largo de la vida de individuos con diferente formación. En segundo lugar, y de acuerdo con el individualismo metodológico imperante en las formulaciones iniciales de la teoria, que implicaba que el origen de los fenómenos sociales se halla en la conducta individual, los retornos sociales en forma de crecimiento económico en que se traduce la inversión individual en educación.

${ }^{6} \mathrm{El}$ modelo de Becker predice que las inversiones en educación en una fase temprana de la vida provocan una función de ingresos cóncava, que se sitúa en un nivel inferior en edades jóvenes y aumenta progresivamente en función de los años, alcanzando un estacionamiento en fases más avanzadas de la vida.
} 
RIS

siones más significativas en formación, en los titulados universitarios en general $y$, en particular, en aquellos que escogen carreras vinculadas a las disciplinas más especializadas y con más años de formación. De este modo, los análisis de Freeman se han centrado en el trabajo de personas con alta cualificación, como ingenieros, abogados o psicólogos. De sus trabajos quizá el más relevante en este ámbito es el referido al mercado de trabajo de los físicos en EE.UU (Freeman, 1975), en el que se parte de la observación de los diversos componentes de este mercado a partir de los años de posguerra. El mercado laboral de físicos en las décadas de 1950 y 1960 se caracterizó por varias convulsiones en sus principales dimensiones. En primer lugar, existieron grandes cambios en la oferta de titulados en física en sus distintas categorías, con una expansión hasta los primeros años 60 y una posterior bajada a partir de esa fecha. Al mismo tiempo, estos cambios estuvieron acompañados de fluctuaciones salariales en el mismo sentidó, primero tendentes al alza y luego con un descenso de salarios. En las mismas fechas también se produjeron variaciones en el gasto público de $\mathrm{I}+\mathrm{D}$, que en los años anteriores se caracterizó por una expansión de las inversiones en investigación de carácter militar en el contexto de la guerra fría, que decrece significativamente a partir de 1960 convirtiéndose en uno de los detonantes de la crisis del mercado de trabajo de los físicos.

A partir de dichas observaciones, Freeman elabora un modelo que comprende una relación de oferta que vincula el número de estudiantes con los sueldos iniciales existentes, y una relación de demanda que depende de los sueldos y los efectos producidos por el comportamiento de los empleadores, incluyendo las inversiones de las empresas y los gastos estatales en I+D. El modelo empleado parte del hecho de que, desde el punto de vista de la oferta, el mercado científico difiere de los demás mercados en la cantidad de tiempo de educación requerido. El comportamiento de la oferta se examina a partir de la denominada 'forma de telaraña', en la que el número de incorporaciones dependería de las cantidades salariales en un momento determinado, mientras que la oferta de titulados se retardaría un período de cuatro o cinco años a partir del cuál influirían los ingresos en la misma. Al considerar que los sueldos iniciales miden de forma aproximada los sueldos que se esperan obtener a lo largo de la vida, debidamente descontados, Freeman se propuso descontar las rentas esperadas, considerando el valor actual de los ingresos como factor explicativo. Los cálculos realizados revelan la existencia de una relación estrecha entre los incentivos salariales y la decisión de estudiar, traducida en el número de matriculaciones. Esto a su vez permitiría realizar un modelo de predicción de la oferta de mano de obra de titulados en las décadas siguientes. El modelo manejado tiene, pues, una estructura recurrente que explica los distintos procesos a lo largo del tiempo en función del cambio de las variables empleadas. Traduciendo sencillamente las posibilidades del modelo, la demanda de físicos por parte de las Universidades, el Estado y las empresas, junto a una escasez de graduados, se traduce en una elevación de las rentas para 
estas ocupaciones, lo cual atrae a su vez a un número incrementado de estudiantes hacia dichos campos. Cuatro o cinco años después estos estudiantes entran en el mercado, empujando hacia abajo los salarios, y provocando de nuevo una redistribución de estudiantes hacia otros campos.

Los análisis de Freeman han sido ampliamente aceptados por la economía al constituir sus resultados empíricos una contrastación de la teoría del capital humano. Para las ocupaciones contempladas en el sistema estadounidense los datos muestran unas tasas considerables de retornos en términos de salarios, o lo que es lo mismo, que los esfuerzos en educación proveen ocupaciones con mayor poder adquisitivo. Así mismo, en la órbita de este enfoque existen numerosos trabajos que, en mayor o menor medida, contrastan y profundizan los supuestos del capital humano en el caso de la ciencia, si bien mayoritariamente estos trabajos se centran en países anglosajones y tienen en cuenta escasamente las características específicas de las cualificaciones o de la estructura ocupacional de la ciencia en sus diversos sectores (Smith y Welch, 1978; Scott, 1979).

\section{La hipótesis de la 'selección'}

Una alternativa en lo referente al funcionamiento de los mercados de trabajo en general son las denominados enfoques de 'la selección' (screening), también llamados credencialistas en ámbitos cercanos a la sociología (Collins, 1971). Tienen en común el plantarse la pregunta no explicada adecuadamente por la corriente del capital humano referida a las imperfecciones en el mercado. Según aquella, el mercado tiene capacidad para absorber a los trabajadores con niveles de formación más elevados, a condición de que los salarios específicos de la formación sean flexibles a la baja (Blaug, 1983). Pero, ¿qué ocurre cuando el mercado no absorbe adecuadamente a las personas con cualificación? Una anomalía empírica del capital humano consiste en que las inversiones en educación no explican los rendimientos posteriores en casos de desempleo o subempleo de personas cualificadas y que, por tanto, al no asegurarse la utilización de recursos humanos, el proceso no se traduciría necesariamente en un mayor nivel de desarrollo económico. La alternativa es poner el énfasis en la demanda de titulados en un mercado de puestos de trabajo partiendo de las preferencias de los empleadores y de la estructura ocupacional que puede absorber a los titulados. Ante la elección de contratar a un trabajador para un puesto determinado, el empleador se enfrenta a un problema de selección. Dadas las dificultades de predecir con precisión los rendimientos del trabajador, se utilizan las credenciales educativas como un procedimiento que reduce los riesgos al suplir la falta de información que se tiene sobre el candidato. La idea central es la creación de 'colas' para puestos de trabajo delimitadas por los requisitos de cualificación, que funcionan como 'vestíbulos' de entrada (Thurow, 1975; Stiglitz, 1975). Dentro estas colas los candidatos compiten en función de la calidad asignada a su formación o credenciales. 
RIS

Así, se introduce aquí el hecho de la influencia de factores como el prestigio de la titulación de procedencia del trabajador junto a los criterios organizativos de la entidad empleadora. Así pues, la contribución de la educación al crecimiento económico sería más bien proporcionar un recurso de selección a los empleadores, que en todo caso son los que ofertan puestos de trabajo y crean una estructura ocupacional y productiva con alta o baja cualificación.

Con el soporte de este enfoque se han realizado algunas investigaciones relevantes, principalmente referidas al funcionamiento de los mercados de trabajo académicos de la educación superior en los EE.UU. Los estudios desde la hipótesis la selección envuelven la noción de que la distribución de científicos en las posiciones del mercado está vinculada con factores institucionales y no tanto con el salario. En la contratación de científicos, por tanto, entran en escena variables relevantes como el tipo de credenciales aportadas por los candidatos y los requisitos exigidos por las instituciones empleadoras. En uno de los escasos estudios empíricos realizados en el ámbito de la ciencia (Niland, 1971), junto a la oferta y demanda de personas con título de $P h D$ se utiliza un perfil de calidad para la producción de doctores y un perfil de calidad para la posición de trabajo de los académicos, haciendo especial diferenciación entre las posiciones docentes e investigadoras en las universidades americanas. De esta forma, se realiza una tabla de movilidad de doctorados entre instituciones de salida y entrada. El análisis de Niland utiliza los supuestos del credencialismo para demostrar cómo las condiciones de cambio del mercado, esto es, la cantidad de oferta y demanda en cada uno de los niveles, afecta al empleo de los titulados. En una oferta al alza de personal científico-técnico las credenciales funcionan como mecanismo de competición para la entrada en los mismos puestos de trabajo. Así, los $P h D$ son situados en una cola basada en la calidad de su educación. Los puestos más elevados se cubren con el personal que reúne las credenciales más deseadas por los empleadores, es decir, los títulos de universidades prestigiosas, desplazando a los otros titulados hacia posiciones de menos calidad. El prestigio y los recursos proporcionados por instituciones concretas podrían determinar el interés de los estudiantes, quienes creen que una titulación de alto prestigio les ayudará a obtener mejores trabajos. Así, un estudiante de $P h D$ con titulación de alto prestigio desplazaría a otro estudiante con un origen menos deseable en una misma ocupación académica para la cuál este último estaría igualmente cualificado. De esta forma, los titulados en instituciones de calidad se distribuirían cada vez más en posiciones de menos calidad.

Basadas también en este enfoque se han obtenido algunas conclusiones generales sobre el funcionamiento del mercado de trabajo científico en los EE.UU (Breneman y Youn, 1988; Atkins, 1974). La primera de ellas es el hecho de que la distribución de las oportunidades en la ciencia está determinada parcialmente por el carácter del progreso tecnológico de la estructura productiva, y también por las orientaciones de instituciones públicas o privadas en lo referente a la obten- 
ción de conocimientos en sus respectivos procesos productivos. Las capacidades de los trabajadores y sus salarios son irrelevantes para determinar el número y clases de posiciones de trabajo existentes y, por tanto, también son irrelevantes para predecir el tamaño del mercado científico. En segundo lugar, los hábitos institucionales influyen en los salarios de los científicos, que generalmente son inflexibles en los puestos de entrada. Por tanto, habilidad, talento o disposición al trabajo tienen un efecto mínimo en los salarios. Por tanto, los trabajadores científicos se inscriben en mercados con distintas condiciones, oportunidades y normas, que son las que rigen los incentivos. Las perspectivas de trabajo están determinadas por la posición relativa en una cola, en la que opera el etiquetaje que se realiza en cada individuo. Los trabajadores más preferidos son los que provienen de las instituciones prestigiosas, que son los que pueden recibir salarios que no son relativos a la productividad o que no corresponden con una tasa de ingresos en función de los años de formación, pero que jerarquizan la posición que ocupan en la organización. Por otra parte, el mercado solventa el problema de los desequilibrios introduciendo más requerimientos de credenciales y más formación adicional.

Vistos los argumentos anteriores, se puede decir que esta corriente se opone a la versión macroeconómica fuerte del capital humano, aunque no contradice la explicación de los tipos privados de rendimiento. La hipótesis de la selección constituye un cinturón protector del núcleo del capital humano (Blaug, 1983) ya que da cuenta de sus inconsistencias, aunque no toca los presupuestos centrales de la teoría. La educación se sigue considerando fundamentalmente como una inversión, esta vez necesaria para competir en un mercado donde las cualificaciones requeridas son cada vez más altas. En todo caso, la utilidad del análisis está en dejar al descubierto los efectos perversos derivados de las imperfecciones que operan en el mercado, aunque no introduce otras variables que profundicen en las condiciones de empleo de los trabajadores científicos.

\section{Los enfoques institucionales}

Durante los años 70 surgen otros tipos de explicaciones que abandonan el modelo que utilizaba las fuerzas económicas como principal factor explicativo del funcionamiento del mercado de trabajo ${ }^{7}$. Mientras que la teoría tradicional trataba la desigualdad social como una evidencia de los logros del individuo en términos de capacidades poseídas y retornos esperados en el mercado, la perspectiva emergente se centra en los amplios mecanismos que operan en la estructura de

\footnotetext{
${ }^{7}$ Sobre los enfoques que incorporan nociones sociológicas en el estudio del mercado de trabajo ver, por ejemplo, Berg (1981) y Gordon (1974).
} 
RIS

desigualdad de las posiciones sociales. El énfasis se pone ahora en la naturaleza múltiple del mercado basándose en observaciones directas de mercados de trabajo en contextos específicos. Michael Piore ha sido uno de los autores que ha abierto una línea teórica centrada en el papel de los contextos organizativos en mercados concretos de trabajo, a la que se suele llamar 'institucionalista' o 'estructural'. Los puntos fuertes del enfoque de Piore están ligados a la noción de la segmentación del mercado y al análisis de los mercados internos de trabajo. En el primer caso la segmentación se refiere a la existencia de un mercado dual de trabajo donde operan reglas distintas. Los principales tipos son el mercado primario, correspondiente a puestos de trabajo de alta cualificación, buenas condiciones de trabajo y procedimientos regulados de acceso y avance, y el mercado secundario, en el que los puestos van asociados a poca cualificación, están peor pagados y dejan un amplio margen para la arbitrariedad (Piore, 1975). Ambos mercados se distinguen por sus requisitos de educación formal, por los tipos de relación entre trabajador y empleador y por las pautas de movilidad y rotación de trabajadores. Pero quizá la aportación más importante desde nuestro punto de vista es la referida a los mercados internos de trabajo, donde se tratan los mecanismos que operan desde la perspectiva de la estructura organizativa en la que se enmarcan mercados concretos (Doeringer y Piore, 1971). Los mercados internos se definen como una unidad administrativa dentro de la cuál el precio y la asignación del trabajo son regidos por un conjunto de normas diferenciadas de las del mercado externo, más cercano a la teoría económica convencional, que estaría sujeto a las 'leyes del mercado'. Las conexiones entre ambos están formadas por 'puertos de entrada y salida' del mercado interno, mientras que los puestos restantes se cubren mediante promoción o transferencia de los trabajadores que ya han conseguido entrar, que están de alguna forma protegidos de las influencias del exterior. Las normas que rigen la asignación de puestos conceden ciertos privilegios a la población laboral interna que no tienen los otros trabajadores y, además, dichas normas no tienen porqué ser explícitas, sino que pueden responder a muy distintos criterios tanto productivos como extra-productivos. En definitiva, el concepto mercado interno combina las nociones procedentes de la economía del trabajo y de la sociología, introduciendo las complejas interacciones entre organizaciones y mercados y realizando un cambio de énfasis desde los atributos de los trabajadores a los atributos de las organizaciones. Para esta corriente la organización es la variable explicativa clave. Cada organización genera sus propios mercados de trabajo e impone un modo burocrático de control social sobre sus empleados, mientras que los mercados de trabajo externos regulan la competencia entre varias organizaciones y condicionan la disponibilidad de trabajadores específicos para los puestos de trabajo que se crean.

Las aplicaciones empíricas de este modelo a las organizaciones científicas han sido llevadas a cabo teniendo en cuenta igualmente el sistema estadounidense de educación superior, en el que existe una gran cantidad de instituciones con diversos 
grados de diferenciación en términos de prestigio y división del trabajo, y que principalmente consisten en universidades orientadas a la docencia y universidades orientadas a la investigación. El grado de diferenciación de las organizaciones académicas provee distintos segmentos de mercado, a saber, para investigación, para enseñanza graduada o posgraduada, o para enseñanza de nivel inferior, entre otras. De este modo, los múltiples mercados barajan posibilidades de múltiples resultados en las carreras científicas, pero la característica más preponderante es que existe una barrera relativamente impermeable entre la enseñanza y la investigación, y sobre todo, en el paso desde la primera a la segunda (Rosenfeld y Jones, 1987; Breneman y Youn, 1986). Este hecho conduce a la evidencia de dos líneas ocupacionales de carácter general, la línea de investigación de alto prestigio y la línea de enseñanza, ambas con distintos mecanismos de promoción. Tal como se verá más adelante, una extensión de este argumento apunta a la existencia de trayectorias internas a partir de la obtención de una posición inicial. Esto es, posiciones de entrada llevan a sistemas de progresión predeterminados. Principalmente la línea de la carrera de investigación puede estar abierta a los logros de productividad científica de acuerdo con el sistema de recompensas de la ciencia, mientras que la línea de enseñanza puede estar determinada principalmente por normas burocráticas, al margen de dicho sistema. De esta forma, dependiendo del tipo de organización concreta existirán distintos criterios para la determinación del logro individual, ya que cada institución puede establecer la distribución de puestos y recompensas de acuerdo con el cumplimiento de sus objetivos específicos ${ }^{8}$.

Como se ha podido observar, la exposición de este conjunto de perspectivas se ha realizado de una forma que va desde presupuestos puramente económicos a otros que introducen progresivamente elementos de observación de carácter social que contribuyen a explicar algunas de las anomalías detectadas en la investigación. A pesar de ello, las líneas de investigación se han mantenido a lo largo de los años sin grandes cambios, principalmente la del capital humano, definiendo áreas de problemas acotadas con contenido propio que observan el mercado de trabajo desde una perspectiva 'externa' que no suele entrar en problemas adyacentes de carácter extraeconómico, si bien sí se han incorporado los avances efectuados

\footnotetext{
${ }^{8}$ Otro aporte interesante desde esta perspectiva es la interacción entre los cambios del mercado de trabajo y los mecanismos de distribución de puestos. Cuando el nivel de la oferta o la demanda cambia sustancialmente, también un número de cambios institucionales puede ocurrir para adaptar la estrategia de la organización a las condiciones de mercado (Smith y Karleski, 1977). Por ejemplo, una fase de expansión de la demanda puede provocar una pérdida de rigidez en los puestos de entrada, produciendo modificaciones en los requisitos de selección de las organizaciones o permitiendo los trasvases a carreras de investigación desde otro tipo de ocupaciones. Al contrario, un periodo de penuria puede aumentar la rigidez tanto en la selección como en los trasvases y, por tanto, impondrá un mayor grado de segmentación de las carreras definidas por parte de las organizaciones.
} 
en líneas complementarias para suavizar los presupuestos teóricos que no se ven corroborados por la realidad observada en la investigación empírica. Es la sociología la que, junto a las corrientes institucionales, se ha ocupado del funcionamiento 'interno' de este mercado, si bien la mayor parte de las investigaciones sociológicas han tenido problemas de investigación y objetivos muy distintos.

\section{Las perspectivas sociológicas}

El sistema de ocupaciones de la ciencia ha sido tratado por diversos enfoques en el campo de la sociología como contexto general en el que opera el mercado de trabajo. Una parte notable de estos estudios tiene en común la influencia de los trabajos pioneros de Robert K. Merton. Además de dotar a la sociología de la ciencia de un campo de problemas específico, las ideas de Merton constituyeron un programa de investigación que dio contenido a la mayor parte de esta especialidad hasta los años 70 e impregnan gran parte de las investigaciones empíricas que posteriormente han perseguido su refutación. De esta forma, una breve referencia a las formulaciones sobre la estructura normativa de la ciencia puede resultar útil como punto inicial para contextualizar adecuadamente los aportes de la sociología, al hilo de las perspectivas que utilizan los presupuestos funcionalistas9.

\section{El reconocimiento como factor estructurador de la institución cientifica}

El eje central de la tradición mertoniana es la consideración de la ciencia como un tipo especial de institución social. El inicial planteamiento del programa mertoniano se encuentra en su conocido ensayo 'La estructura normativa de la ciencia' (Merton, 1977a), en el que se sostiene que la ciencia está marcada por un ethos específico, constituido por una serie de normas y valores comunes al

\footnotetext{
${ }^{9}$ Se han dejado al margen un importante grupo de estudios que tratan las especificidades de la ciencia como profesión (Ben David, 1972; Polanyi, 1962; Miller, 1991), así como la abundante literatura sobre la profesión académica o los estudios socio-psicológicos sobre las carreras científicas. Compilaciones de ambas perspectivas pueden verse respectivamente en Clark (1987) y Eiduson y Beckman (1973).

${ }^{10}$ Las normas que configuran el ethos están expresadas en forma de prescripciones, proscripciones, preferencias y permisos, y se legitiman en términos de los valores de la institución de acuerdo con el cometido principal de la ciencia: la extensión del conocimiento científico certificado. A juicio de Merton, aunque este sistema normativo no ha sido codificado, puede ser inferido a partir de las declaraciones de los científicos, de sus escritos sobre la ciencia, así como de la observación de su conducta. Además, este conjunto de normas y valores se refuerza mediante un conjunto especial de sanciones y recompensas, y se conciben como preceptos técnicos que garantizan el progreso del conocimiento certificado. Una discusión sobre el programa mertoniano puede verse en Torres (1995). Los trabajos aquí citados se encuentran recopilados en Merton (1977).
} 
comportamiento de los científicos, que permite que esta actividad se haya consolidado a lo largo de la historia en una institución diferenciada y duradera ${ }^{10}$. En la original formulación de la estructura normativa Merton propuso cuatro normas fundamentales, que se resumen en la conocida regla de los CUDEOS: Comunismo, Universalismo, Desinterés y Escepticismo Organizado. De ellas, quizá sea la norma del universalismo la que ha guiado la mayor parte de los estudios empíricos encaminados a contrastar dicha estructura normativa, y es la norma que tiene mayor relevancia en lo relacionado con el mercado de trabajo científico. El universalismo se refiere al requerimiento de que las contribuciones de los científicos sean juzgadas de acuerdo con criterios impersonales preestablecidos. Los atributos sociales como religión, raza o sexo, no deben contar en la evaluación del trabajo científico y, por tanto, los científicos sólo deben ser recompensados por sus contribuciones a la ciencia. La otra cara del universalismo en lo referido al funcionamiento del sistema de ocupaciones de la ciencia es la meritocracia, esto es, el acceso a los puestos de trabajo de la ciencia y la movilidad a través de su estructura ocupacional según el valor de las contribuciones científicas contrastadas y sancionadas públicamente, como se verá más adelante.

Posteriormente, la formulación inicial fue replanteada y matizada en otra serie de trabajos que complementaban la perspectiva normativista con otra interpretación utilitaria que daba pie a una explicación más abierta de los motivos que mueven el comportamiento de los científicos. En concreto, en el trabajo sobre las disputas en las prioridades de los descubrimientos se examina la particular orientación de la conducta científica a la producción de conocimientos originales, y su relación con el sistema de recompensas de la ciencia. Para Merton, dentro del orden científico existe un sistema de intercambio basado en la concesión de recompensas, que consiste en la aportación de conocimientos que los colegas valoran como útil y original, y por la cuál se reciben una serie de tributos. Las recompensas se ejecutan a través del reconocimiento, que puede adoptar distintas formas. Una se realiza de modo informal y está basada en el reconocimiento de los colegas, mientras que otra se institucionaliza en recompensas que se otorgan de un modo más formalizado, como los premios, las distinciones y los cargos honoríficos. El sistema institucionalizado de recompensas tiene su origen en la existencia de las normas de la ciencia, que son las que permitieron la introducción de este particular mecanismo de intercambio. De esta forma, el sistema de trueque de aportaciones científicas por reconocimiento contribuye al reforzamiento de las normas y valores morales de la ciencia y, con ello, a la consecución de los objetivos de la institución. Aunque el esquema de Merton responde al enfoque funcionalista, la noción del intercambio deja abierta la posibilidad de que existan comportamientos alternativos a los establecidos por las normas. En posteriores estudios el mismo autor examina cómo variables adicionales interactúan con los factores indicados en el paradigma, mostrando que en la ciencia, al igual que en otras instituciones, existe un contraste problemático entre las expectativas 
RIS

normativas y el comportamiento real. Ejemplos de ello son la acumulación de recompensas por ciertos científicos a partir del prestigio, el llamado 'efecto Mateo', o la posibilidad de que existan conductas desviadas de las normas.

El interés de este conjunto de estudios para el análisis sociológico del mercado de trabajo en la ciencia está en la utilización de la ocupación como uno de los elementos constitutivos de la estructura social. La posición ocupacional o puesto de trabajo se ha utilizado como una forma de operacionalizar las desigualdades y observar los procesos que operan en ellas, como es el reconocimiento a partir de las contribuciones realizadas, y a la vez como el punto desde el que se alcanzan distintos grados de estatus, recursos y poder en la ciencia. Los estudios provenientes de esta perspectiva se sitúan en la dicotomía universalismo-particularismo a partir de la constatación de que la estructura social de la ciencia sea o no el resultado de la adjudicación de recompensas diferenciales en función de los méritos reălizados. En ellos se pueden aislar ciertos elementos que han sido empleados recurrentemente tanto en la observación de los procesos de estratificación como en el estudio de la incorporación y movilidad profesional en la ciencia. El primero es la 'producción científica', las publicaciones, que se entienden como el vehículo por el que se realizan las contribuciones originales y certificadas al conocimiento científico. Para los estudiosos de la estratificación aparece como el principal elemento sobre el que es posible establecer objetivamente el mérito individual. El segundo factor es el 'reconocimiento', que constituye el proceso de validación de las contribuciones realizadas por un científico por parte de los miembros de la comunidad. Es en sí mismo una recompensa y, a la vez, es el mecanismo por el que se establecen la mayor parte de las recompensas en la ciencia. El tercero lo constituye la 'posición organizacional', entendida como la situación relativa del científico en el seno de la institución, normalmente basada en términos de prestigio de la organización y del puesto que se ocupa en ella. Estos tres elementos, que podemos denominar 'internos', han sido empleados profusamente haciendo distinto énfasis en el papel que juegan en los procesos de estratificación en la ciencia. Los estudios realizados pueden clasificarse por la posición que ocupan las contribuciones científicas en el análisis, en tanto que variable explicativa o explicada de cada modelo.

En el primer caso, adoptado por los enfoques más estrictamente funcionalistas, las publicaciones actúan como la base sobre la que se realiza el reconocimiento de la actividad científica, y sobre la cuál a su vez funciona el sistema de recompensas de la ciencia, una de las cuáles es la posición del científico. De este modo, la cuestión central en los estudios desde el paradigma mertoniano se ha dirigido a observar cómo las investigaciones de calidad realizadas por los científicos se correlacionan con las recompensas que reciben. Por ejemplo, el intercambio de aportaciones científicas por reconocimiento ha sido utilizado habitualmente por los sociólogos funcionalistas en los estudios empíricos sobre la institución científica. Estudios tempranos son los referidos al funcionamiento de la comunidad científica (Hagstrom, 1965), o los trabajos centrados en la observación de las 
carreras profesionales desde el punto de vista del grado y el tipo de reconocimiento que es necesario para el progreso profesional en el seno de una organización (Glaser, 1967). Más recientemente, uno de los estudios relevantes es el realizado por los hermanos Cole en grupos de científicos naturales (Cole y Cole, 1973), que utiliza la producción científica como el eje sobre el que operan los procesos de evaluación. Así, el número de citas recogidas de los trabajos de un científico es indicador de la relevancia y calidad de las contribuciones al conocimiento. Además, se establece que el reconocimiento obtenido formalmente a través de las citas constituye el principal motivador de la conducta de los científicos. Las citas proveen, por tanto, el elemento sobre el cuál es pertinente realizar una valoración de las contribuciones al progreso científico y permite establecer un elemento objetivo para la asignación de recompensas.

Los resultados de estos estudios muestran cómo los procesos de evaluación se traducen en una estratificación de la institución científica sobre criterios de mérito, asignando mayor cuota de recompensas a los científicos con mayores contribuciones, tanto en cargos honoríficos como en posiciones laborales. Sin embargo, a pesar del alto grado de universalismo, no existe un estricto cumplimiento de la norma. Hay factores que afectan negativamente al sistema de evaluación citado, como es el prestigio que los departamentos de formación tienen en el reconocimiento. La ubicación en las fases iniciales de la carrera en departamentos de mayor prestigio se traduce en ventajas acumulativas que proveen mayores recursos para investigar, mayor visibilidad y mayor estatus, lo que produce la obtención de distintas posiciones en la estructura social. De esta forma, existe un efecto similar al efecto Mateo en el que interviene el etiquetaje, por lo que la posición inicial de partida se confirma en el éxito posterior. No obstante, a pesar de los efectos distorsionantes, se concluye que ninguna otra institución presenta una base tan universalista para la estratificación y que, además, la distribución desigual de recompensas no genera conflictos y presenta un alto grado de consenso.

Otra aportación relevante en la estela del programa mertoniano son los estudios de Gastón centrados en grupos de físicos y biólogos (Gaston, 1978). Sin embargo, aceptando la vigencia del sistema de recompensas, estos estudios abundan en los diversos mecanismos que provocan efectos perversos. Gastón desarrolla extensamente el principio del efecto Mateo en otras variantes que pueden ocurrir en el seno de la institución buscando elementos que interfieren en el universalismo ${ }^{11}$ y muestra cómo las prácticas de reconocimiento pueden verse afectadas por

\footnotetext{
"Algunos de los que pueden citarse son el caso del científico situado en una institución periférica, que con el paso del tiempo obtiene cada vez menos oportunidades para investigar (Efecto 'Podunk'), o el caso de los científicos en instituciones de alto prestigio, que alcanzan un reconocimiento mayor del que sus contribuciones merece (Efecto 'Knudop'). Por otra parte, Gastón cita otra serie de factores de ámbito macrosocial que afectan al reconocimiento, como son los distintos grados de consenso
} 
RIS

REVISTA INTERNACIONAL DE SOCIOLOGIA

№ 32, Mayo-Agosto, 2002

MANUEL FERNÁNDEZ ESQUINAS

múltiples factores, existiendo notables diferencias en el cumplimiento de los principios universalistas, aunque este autor también es partidario de la vigencia del universalismo en las pautas de estratificación de la ciencia. Quizá ha sido Zuckerman la autora que, dentro del programa normativista, ha prestado más atención a los modos en que variables adicionales interactúan con el universalismo. En el trabajo sobre pautas de evaluación instucionalizada (Merton y Zuckerman, 1971) se analiza la influencia de diversos factores sociales en la evaluación de los trabajos científicos y en el acceso a los canales de comunicación, mostrando cómo el prestigio actúa en la sobrevaloración de trabajos de individuos conocidos, o cómo el consenso cognitivo provoca la aceptación preferente de trabajos adaptados a las posturas imperantes en las disciplinas científicas. Por otra parte, esta autora también ha desarrollado una serie de trabajos en los que se demuestra la constante de las desigualdades en la comunidad científica, como el estuidio sobre la estratificación de ciencia americana (Zuckerman, 1970) o el estudio de los premios nobel (Zuckerman, 1967) en los que observa el reclutamiento selectivo y la socialización diferencial que tiene lugar entre universidades y científicos de élite. En definitiva, los trabajos elaborados por la corriente funcionalista produjeron una serie de anomalías que dieron paso a la introducción de otros enfoques en el estudio de la estratificación científica a partir de los años 70 que, si bien no suponen un cambio drástico de paradigma, sí introducen nuevos elementos en la metodología de observación de las carreras de los científicos.

\section{La influencia de la adscripción}

El recurso al reconocimiento mediante las citas suele presentar el problema de que su utilización sólo es factible cuando existe producción científica de calidad que sea susceptible de ser evaluada. De esta forma, su empleo no da cuenta de los diversos factores influyentes en el logro de méritos en los que posteriormente se basa el reconocimiento. Así, estudios posteriores realizan una reubicación de los elementos utilizados en el análisis funcionalista. Mientras que el enfoque anterior sugiere que los departamentos más prestigiosos seleccionan a sus miembros entre los científicos más productivos, mostrando cómo la productividad tiene un efecto en los puestos académicos, la versión alternativa traslada el énfasis a los factores que inciden en la productividad científica como elemento explicativo primigenio previo al proceso del reconocimiento. $Y$ uno de los elementos principales lo constituye el tipo de institución científica de procedencia y las ocupaciones de inicio en la estructura ocupacional de la ciencia.

en las disciplinas científicas que pueden provocar conflictos entre teorias o enfoques alternativos, o la situación organizativa de la investigación en cada país, con formas particulares de distribución de recompensas. 
En este enfoque se sitúan algunos de los estudios empíricos realizados por Diana Crane entre grupos de científicos en los Estados Unidos durante la década de los 60, en los que se centra en la observación de los efectos adscriptivos tanto en la producción científica como en el reconocimiento. Para ello utiliza una serie de indicadores institucionales basados en criterios de prestigio (prestigio de los lugares de trabajo y procedencia académica, así como del sponsor académico), junto a índices de productividad (contribución de los investigadores según años de antigüedad desde la finalización del doctorado) e índices del reconocimiento (tipos de recompensas honoríficas recibidas, como premios, becas prestigiosas o pertenencia a asociaciones científicas) (Crane, 1965). Su estudio muestra cómo los graduados en universidades prestigiosas tienen más posibilidades de tener alta productividad, e incluso en universidades de alto prestigio se sigue manteniendo mayor productividad en aquellos provenientes de instituciones reconocidas: De la misma forma, científicos que han tenido patrocinadores eminentes tienden a ser más productivos. Por otra parte se observa que la procedencia además influye en la obtención de un puesto de trabajo en universidades prestigiosas. El reconocimiento de la actividad del científico se ve afectado por la evaluación de su trabajo, ya que ésta se basa en la producción, pero también por el tipo de posición ocupada en la institución empleadora, que funciona como un 'halo' que dota de mayor visibilidad e importancia entre los colegas a los trabajos provenientes de instituciones de prestigio. La interpretación que propone la autora de este hecho está basada en los procedimientos de selección de estudiantes, en los que, a modo de hipótesis, introduce la cuestión del talento. Los mejores estudiantes son seleccionados por las mejores escuelas, y los mejores de ellos son seleccionados por los mejores científicos. De este selecto grupo es de donde proceden los científicos que a su vez serán más productivos en subsiguientes generaciones ${ }^{12}$.

Otros estudios abundan en las tesis de la adscripción institucional buscando las variables utilizadas por los departamentos para elegir a sus miembros, incluida la producción científica, utilizando datos de carácter longitudinal en la carrera de los científicos. En una investigación sobre la posición inicial en el sistema académico

\footnotetext{
${ }^{12}$ En posteriores estudios (Crane, 1969;1971) se confirma la hipótesis de la adscripción mediante el examen de las características de científicos en departamentos de alto prestigio, sugiriendo que los elementos utilizados por los responsables del reclutamiento para predecir las realizaciones de los jóvenes científicos en el futuro atienden más a la institución de origen que a las realizaciones previas. A pesar de existir un compromiso normativo con los criterios del universalismo, éstos no son utilizados en la práctica. El origen de los doctorandos tiene más influencia en la selección para una posición prestigiosa que la productividad cientifica, por lo cuál el sistema de recompensas debe ser explicado por las reglas de la adscripción en el sentido de que las mayores universidades proveen mejores oportunidades para el contacto con científicos eminentes y para la realización de investigaciones relevantes.
} 
de una muestra de bioquímicos (Long et al., 1979), se contrasta igualmente la hipótesis de que la productividad previa al empleo tiene un efecto insignificante en el prestigio de la posición inicial de un científico, introduciendo otras variables a modo de predictores del logro científico. La observación de la naturaleza de la distribución de recompensas se realiza comparando aquellos factores que determinan la distribución de trabajos con los que determinan la producción científica en la vida del individuo. Si unos y otros coinciden, sería posible establecer una distribución de trabajos basada en criterios de mérito. Sin embargo, los análisis muestran que existe una correlación entre los grados de prestigio de los departamentos de origen y los departamentos en los que se obtiene el primer trabajo, incluso cuando esto tiene escaso efecto en la productividad futura, mientras que existe muy poca relación entre productividad anterior y primer trabajo. Por otra parte, se detecta que el origen tiene un pequeño efecto en el recuento pošterior de publicaciones científicas. El mejor predictor de la productividad se encuentra en la existencia de productividad anterior al primer empleo. Por tanto, parece que en las pautas de selección se tiende a ignorar este mayor predictor de la capacidad futura. Más aún, en un posterior estudio (Long y McGinnis, 1981) se muestra que la actividad de la investigación científica es determinada por los departamentos en los que un científico se adscribe más que al contrario, y que la actividad de los científicos viene a reproducir la actividad de aquellos con los que se asocia al comienzo de la carrera. El estudio concluye así que el acceso de los científicos a la primera posición está regido por procesos adscriptivos o reglas particularistas.

En el debate sobre logros versus adscripción algunos estudios ponen mayor énfasis en otra fuente de efectos, como son la influencia de los patrocinadores 0 sponsor en las carreras científicas. Aunque ya había sido tratado por los estudios citados, y anteriormente por los funcionalistas (Hargens y Hagstrom, 1967), Reskin elabora un modelo que establece la influencia de este agente en la variación en las carreras de los científicos no explicadas suficientemente por el prestigio de los departamentos de formación. En la formación de estudiantes los patrocinadores trasmiten las capacidades profesionales, lo cual puede influir en las realizaciones científicas de sus tutelados. Pero, por otra parte, los sponsor adscriben al estudiante a un rango inicial de estatus en el sistema de la ciencia, ya que puede existir una extensión o identificación del estatus del patrocinador con el del patrocinado, lo cual provee de mayores posibilidades de investigación, nominaciones o recomendaciones, e incrementa la visibilidad del trabajo científico. La comprobación de esta hipótesis se realiza en una investigación sobre químicos (Reskin, 1979) en la que se elabora un modelo que pone en relación las características del patrocinador, medidas tanto en producción científica como en 'eminencia' o prestigio, con los subsiguientes logros en la carrera del científico a través de los trabajos obtenidos y la productividad científica. Los resultados en la combinación de las diferentes variables permiten concluir que el tipo de patrocinador, junto al tipo de departamento, es crucial para las carreras de los científicos. Los análisis muestran una 
correlación entre la colaboración previa con un patrocinador de prestigio y una mayor productividad. Además, las características de los patrocinadores correlacionan con el prestigio de las primeras posiciones ocupadas y siguen persistiendo en las subsiguientes ocupaciones.

En definitiva, el conjunto de estudios agrupados en esta sección concluyen que los logros científicos no parecen constituir el escenario perfecto para una competición en un sistema de oportunidades abierto, y que la estratificación en la ciencia no es resultado de un sistema de recompensas competitivo en el que cada miembro es evaluado sobre la base de su contribución al conocimiento. Así, los factores extra-universalistas tienen una notable presencia en el mundo de la ciencia académica, si bien hay que aclarar, aunque estos autores no lo hagan, que no son factores extra-académicos, sino que se encuentran en el modo de funcionamiento tradicional de este tipo de ciencia desde la transformación de las universidades como corporaciones que tienen entre sus principales cometidos la producción de conocimientos científicos certificados ${ }^{13}$.

Por último, y aunque no se integra exactamente en ninguna de las corrientes anteriores, es conveniente citar un estudio que, si bien tiene ya cierta antigüedad, es relativamente excepcional al integrar en él gran parte de la complejidad social que subyace al mercado de trabajo, en este caso el académico (Caplow y McGee, 1958). La importancia de este trabajo radica en que tiene en consideración tanto los factores individuales como organizativos cuando se estudia la entrada y movilidad en las universidades norteamericanas y, sobre todo, su relevancia se encuentra en la original definición de su unidad de análisis. Ésta la constituye el proceso de vacante-reemplazamiento de los puestos de trabajo, es decir, la forma en que un puesto se crea o se desocupa, y la forma en que se cubre por un nuevo trabajador. Con esta definición es posible, pues, delimitar la importancia que tanto los criterios de la institución como de los seleccionadores tienen en la forma de

\footnotetext{
${ }^{13} \mathrm{La}$ evidencia dejada por estas investigaciones da paso a estudios en los que la observación de las desigualdades se amplía a elementos sociales o de carácter extracientífico, fundamentalmente el sexo y la raza, que ya fueron iniciados por las aplicaciones empíricas de la corriente funcionalista (Zuckerman y Cole, 1975). Numerosas investigaciones han abundado en los diferentes procesos que operan en la desigualdad de la ciencia a partir de características adquiridas o adscritas. Una de las recientes es Debackere y Rappa (1995). Una revisión puede verse en Long y Fox (1995).

De hecho, el estudio de las desigualdades sociales a partir de estos factores ha sido uno de escasos campos en que las investigaciones empíricas han seguido teniendo cierta presencia después del declive del paradigma funcionalista y del surgimiento de las sociologías del conocimiento científico en los años 70 . Quizá el problema de investigación más relevante es el referido a 'mujer y ciencia' debido a que recibe las aportaciones de la sociología del género aplicada en otros ámbitos y, sobre todo, porque esta línea de investigación ha contado con apoyo político y financiación al contemplarse en planes de I+D tanto nacionales como internaciones Ver, por ejemplo, Shenhav y Haberfeld (1988).
} 
RIS

contratación y en las características de los trabajadores que finalmente ocupan los puestos. El trabajo de Caplow y McGee se ha convertido en un clásico del mercado de trabajo académico, con algunas continuaciones importantes que abundan en los cambios de las universidades americanas (Burke, 1988), si bien su metodología ha sido escasamente empleada por los sociólogos de la ciencia.

\section{El ámbito de la ciencia industrial}

Los estudios hasta ahora citados habitualmente se han circunscrito al mundo académico, que era el mundo que constituía el reino del conocimiento público en los años en los que se desarrolló el programa de investigación funcionalista y sus secuelas. No obstante, tanto en la órbita de la corriente funcionalista como posteriormente desde otros enfoques, existe una línea de investigación que se ocupa de los científicos en la industria tratando problemas referentes a su sistema de valores y a su estructura de ocupaciones.

El empleo de investigadores por parte de la empresa comenzó a analizarse desde la asunción de que existe una frontera cultural entre el mundo empresarial y el mundo científico, al existir en ellos sistemas de valores contrapuestos. Así, los primeros trabajos desde la corriente funcionalista tratan el asunto como el problema de la acomodación de los científicos en el sector industrial. Una de las primeras síntesis es el clásico trabajo de Kornhauser en el que la industria era vista como un entorno organizativo que prestaba más énfasis a los valores económicos y administrativos, en contraposición a los valores presentes en la ciencia (Kornhauser, 1963). Esta distinta configuración social daba lugar a una serie de tensiones entre los valores de los trabajadores científicos en la industria y los valores que priman en las organizaciones que los empleaban. En primer lugar, existe un conflicto de objetivos, dado que los científicos persiguen trabajar en campos de investigación susceptibles de hacer contribuciones relevantes a la ciencia intercambiables por reconocimiento, mientras que los directivos de la industria quieren que los científicos se concentren en problemas cuyos resultados den lugar a un beneficio. En segundo lugar, existe un conflicto de control sobre la situación de trabajo, al buscar los científicos asumir la organización y ritmo de finalización de los proyectos, mientras que desde la organización se buscan grupos de trabajo que realicen su labor de acuerdo con las prioridades de la empresa, con recursos y tiempo limitados. En tercer lugar, también existe un conflicto de incentivos, dado que los científicos prefieren recompensas relacionadas con sus necesidades profesionales, tales como autonomía o tiempo libre, mientras que las recompensas en el mundo de los negocios se basan más en la promoción en la jerarquía organizativa y en incentivos económicos. Por último, existe un conflicto de responsabilidad en la utilización de los productos de la investigación: los profesionales de la ciencia normalmente realizan ciertas consideraciones éticas 
sobre la finalidad de su trabajo mientras que las consideraciones de las compañías responden a razones comerciales.

Estos conflictos han sido tratados por desarrollos posteriores en la sociología de la ciencia y de las organizaciones, existiendo literatura que desmiente el enfoque funcionalista, señalándose que el trabajo científico tiene lugar en una variedad de marcos institucionales. Existe evidencia de que las estructuras normativas varían profundamente, con algunos estudios que muestran cómo los científicos que ingresan en la industria contemplan su trabajo desde una óptica instrumental y se adaptan fácilmente a los valores de los negocios (Box y Cortgrove, 1971; Barley, 1989). Sin embargo, los argumentos desde la óptica funcionalista pusieron de manifiesto la existencia de distintas lógicas de actuación en dos contextos organizacionales que requieren distintas pautas de adaptación en los trabajadores empleados por ellos, lo que sigue siendo relevante debido a que una fuente de conflictos radica en la mayor presencia de una ideología profesional en los científicos, que entra en contradicción con las lógicas de la producción industrial ${ }^{14}$. De hecho, una parcela importante de la moderna literatura dedicada a la gestión de recursos humanos en la empresa trabaja en la resolución de problemas específicos derivados del empleo de profesionales altamente cualificados, como son los científicos ${ }^{15}$. Las organizaciones que emplean científicos e ingenieros se enfrentan al dilema de establecer un sistema de recompensas que sea estimulante en términos profesionales y a la vez eficaz para la organización. Este problema sigue estando basado en el hecho de que los especialistas llevan a la organización actitudes y aspiraciones profesionales que pueden estar en conflicto con los requerimientos del trabajo. Un conocido ejemplo para la resolución de los conflictos que plantean las expectativas profesionales de estos trabajadores es el de 'la estructura dual' de carreras ocupacionales, que diferencia entre los incentivos de tipo profesional y las recompensas que se ofrecen, distinguiendo entre las aspiraciones de los científicos e ingenieros de aquellos dedicados a tareas de gestión y dirección (Allen y Katz, 1986). En suma, a pesar de que sea conveniente ver los términos del debate desde una perspectiva distinta a la empleada por la sociología funcionalista ${ }^{16}$, la evidencia empírica nos muestra que existe cierto ethos profesional que está presente en el colectivo de científicos, en parte en virtud a los mecanismos

\footnotetext{
${ }^{14} \mathrm{Un}$ análisis de las contradicciones entre ciencia y producción desde una perspectiva económica puede verse en Vence (1992).

${ }^{15}$ Un ejemplo de estos enfoques puede verse en la recopilación de Gómez Mejía y Lawless (1992).

${ }^{16}$ No se puede argumentar que todos los científicos están socializados en el sistema de valores de la ciencia, y tampoco se pueden inferir motivos y valores del comportamiento que se observa. En este sentido, Box y Cortgrove indican que hay que considerar la posibilidad de que la conformidad con los valores de la ciencia pueda ser sólo el reconocimiento de las reglas del juego. A saber, si alguien está inmerso en la vida académica se ve en la obligación de aceptar las reglas de la academia.
} 
de socialización en la ciencia, y que ésta es una posibilidad de explicación de la existencia de tensiones y conflictos entre los ideales de la investigación y la forma de control ejercido por la industria.

\section{BALANCE DE LA INVESTIGACIÓN SOBRE EL MERCADO DE TRABAJO Y EL SISTEMA DE OCUPACIONES CIENTÍFICAS}

Difícilmente se encuentran en la literatura análisis que traten de forma comprehensiva los diversos condicionamientos que configuran el conjunto de ocupaciones de la ciencia y que especifiquen el modo de funcionamiento del mercado de trabajadores científicos. Los estudios han estado frecuentemente fragmentados por los programas de investigación empleados por los distintos enfoques, con presupuestos de partida y objetivos distintos, aunque no necesariamente contradictorios. Además de la divergencia teórica, una limitación importante de buena parte de las investigaciones empíricas consiste en su extensión y en los marcos de referencia a los que se circunscriben. Numerosos estudios se han basado en muestras de tamaño reducido en centros o especialidades concretas, con poblaciones de referencia que dejan al margen a la mayoría de especialidades 0 instituciones, y en bastantes casos seleccionando a científicos en departamentos de 'élite'. De esta forma, ante la imposibilidad de observar la amplitud de los procesos descritos resulta difícil generalizar conclusiones al conjunto de la institución científica, sobre todo teniendo en cuenta que la mayoría se centran en el modelo norteamericano.

Los estudios provenientes de la economía del trabajo parten de la consideración del mercado científico como otro tipo de mercado, diferenciando sólo en el nivel de la cualificación requerida y sin tomar en consideración las especificidades del sistema social de la ciencia. En concreto, las teorías del capital humano presentan la limitación de aplicar una excesiva racionalidad de tipo utilitario al establecer los determinantes de la conducta individual en la búsqueda del interés económico. Al partir del supuesto de que la elección del trabajador para decidir si trabaja o no es una elección entre la renta que le supone el trabajo y el ocio de no trabajar, se equipara al trabajo con otro tipo de mercancía haciéndolo depender de su precio. La elección de trabajar o no es así función del precio del trabajo, es decir, del beneficio que se consigue al realizar el esfuerzo, comparado con el beneficio que se deja de conseguir al no realizarlo, existiendo de este modo un efecto de la renta y un efecto de sustitución en función del precio del trabajo. Subyacente a este modelo se encuentra la idea de que el trabajo es solamente un medio para conseguir un fin, la ganancia económica, pero en sí mismo es visto desde un punto de vista negativo. Por tanto, siempre será preferible el ocio al trabajo a no ser que exista un incentivo pecuniario que lo contrarreste. Esta concepción del trabajo deja fuera cualquier papel sustantivo en el trabajo de 
investigación, como pueden ser las motivaciones de tipo vocacional. Si bien el nivel salarial de la ocupación científica puede tener influencias en la decisión de los trabajadores, esta perspectiva reduccionista tiene notables limitaciones, sobre todo en los mercados más alejados del modelo del mercado perfecto, es decir, casi todos ellos al margen de los Estados Unidos. Por otra parte, la atención prestada al individuo como unidad de análisis abstrae los efectos de la estructura social y los entramados institucionales que pueden afectar las carreras de los científicos, tratando los mercados de trabajo como si funcionaran en el 'vacío'.

Aunque ello ha sido señalado por los enfoques institucionales, éstos también presentan otras limitaciones. De un lado, la mayor parte de los estudios empíricos se han realizado sobre mercados de trabajo ajenos a la ciencia y las experiencias de investigación de mercados científicos se centran igualmente en ejemplos limitados. De otro lado, se echa en falta, si no la presencia de un conjunto de valores específico distinto al de la racionalidad económica, sí las particularidades organizativas de la ciencia derivadas de los objetivos de la institución, así como la institucionalización de unos controles de calidad específicos y de una forma de recompensas muy especial que difícilmente tiene equivalentes en otra institución. No obstante, la limitación de este enfoque consiste más bien en la ausencia de investigación empírica continuada, a pesar de que las nociones de segmentación y mercado interno se adaptan a la estructura ocupacional de la ciencia y constituyen una buena herramienta de análisis al incorporar la influencia que las condiciones del mercado pueden tener en el funcionamiento de la institución científica, como se verá más adelante.

Los estudios empíricos realizados desde perspectivas sociológicas han dejado constancia de que en esta institución operan notables niveles de desigualdad y discriminación, al igual que en otras organizaciones sociales, y que la base que las sustenta no tiene porqué ser de carácter especial. Pero para los objetivos que aquí se contemplan los estudios sociológicos citados presentan otra serie de dificultades referidas a las herramientas de análisis utilizadas en la medida de la estratificación, en concreto, el concepto de universalismo y los indicadores de productividad, reconocimiento y prestigio académico.

Sobre el concepto de universalismo, su formulación responde principalmente a un tipo de ciencia, el practicado en las instituciones académicas en las que se da primacía al conocimiento público contrastado con los pares de la profesión a través del sistema de comunicaciones de la ciencia. Es relevante, pues, tener en cuenta que su opuesto, el particularismo, lo es teniendo en cuenta el principio de publicidad, debido a que en otros tipos de ciencia no se valoran de la misma forma las recompensas por las contribuciones realizadas, o bien hay otras formas de recompensas para resultados científicos no sujetos en primera instancia al principio de la publicidad. En suma, establecer esta clase de universalismo como opuesto al particularismo, es decir, definir lo que no se corresponde con él como particularismo, tiene que ver con la noción ideológica de la ciencia que sustenta 
RIS

REVISTA INTERNACIONAL DE SOCIOLOCIA

No 32, Mayo-Agosto, 2002

dicho argumento. Y la ideología de la ciencia que subyace aquí es la ideología académica (Mulkay, 1976), la que sostiene que el conocimiento público certificado por los pares, el que es evaluado por los propios miembros de la comunidad académica, es el conocimiento válido en la actividad científica, $\mathrm{o}$ al menos más válido que el que está contrastado por el uso en el ámbito político, tecnológico, social o económico. No quiere esto decir que el conocimiento público no sea válido, sino que pueden existir otras nociones ideológicas que consideran otros tipos de certificación de los conocimientos científicos de forma complementaria $o$ alternativa a las publicaciones, como son las patentes, los informes de investigación o las aplicaciones tecnológicas a procesos industriales, entre otros muchos. Así, considerar al universalismo científico de corte académico como vara de medida de la meritocracia supone seguir aceptando las prescripciones normativas del funcionalismo, prescripciones que además se consideran beneficiosas o funcionales para el desarrollo de la ciencia. Y desde un punto de vista de la investigación científica el posible beneficio debe ser una cuestión de investigación empírica cuyos resultados se evalúan a posteriori y teniendo en cuenta el marco valorativo e ideológico desde el que se realiza la evaluación. Por consiguiente, el supuesto del universalismo basado en la producción científica pierde relevancia en aquellas instituciones que no priman directamente este hecho, y que se corresponden frecuentemente con las universidades más vinculadas a la docencia, con los centros de investigación gubernamentales y, sobre todo, con la industria. Algunas de las críticas a los indicadores utilizados en los estudios sociológicos bajo esta rúbrica se enmarcan en el problema de fondo de la meritocracia, a saber: la utilización casi exclusiva del indicador de la productividad como realización científica, así como las citas como indicador de reconocimiento, lo que presenta numerosas inconsistencias. Considerar las publicaciones como la única actividad relevante puede ser una concepción reduccionista de la actividad del científico. Si bien la generación de conocimientos es una tarea esencial, en algunos entornos tienen especial importancia investigaciones no dirigidas a publicación, tareas de docencia, de formación de nuevos científicos o de gestión de la investigación, cuestiones que igualmente pueden considerarse como logros $\mathrm{y}$, por tanto, como influyentes en la estructuración ocupacional ${ }^{17}$.

\footnotetext{
${ }^{17}$ Quizá la medida de la productividad sea adecuada en aquellos científicos que dedican su trabajo por entero a la investigación, pero no parece ser un criterio válido para todas las ocupaciones en el mundo académico. De hecho, la mayoría de las personas calificadas como científicos o investigadores raramente se dedican por completo a investigación, siendo un hecho constatado que la asunción de responsabilidades de forma paralela a la promoción profesional detrae tiempo de tareas de investigación a favor de las tareas de gestión, captación de recursos y relaciones públicas con el exterior (Ziman, 1989).
} 
Por otra parte, la utilización de las citas como forma de reconocimiento presenta dificultades de aplicación en aquellos individuos que tanto por edad como por otros motivos no poseen publicaciones. El recurso a la productividad científica sólo sería útil a partir de ciertos momentos en la vida de los científicos, ya que ésta empieza a surgir en una etapa de la vida profesional, que es distinta en cualquier especialidad e, incluso, en cualquier área de problemas. Con anterioridad a esta producción existen otros procesos de adscripción basados en factores distintos de la producción. Aunque ello ha sido constatado ampliamente por la corriente institucionalista, sin embargo sus estudios quedan muy lejos de demostrar cómo operan los distintos tipos de factores sociales en la producción científica, como, por ejemplo, elegir un problema de investigación 'fácil' o 'difícil' o comenzar en una línea por propia elección o por imposición ${ }^{18}$. Si bien es adecuado seguir utilizando los productos científicos como elemento fundamental para la observación y la valoración de los logros en la ciencia, sí se puede decir que éstos corresponden a un sistema de organización científica específico, o a la extensión de la ideología de la ciencia imperante en un momento y un tipo organizativo específicos. La pregunta por la relevancia de este sistema de recompensas, o por su extensión en distintas disciplinas e instituciones, posiblemente no se pueda contestar sin análisis comparativos sistemáticos, siendo cada uno de los factores señalados más o menos relevante según la institución concreta en la que operen. Así mismo, el conjunto de estudios posteriores bajo la rúbrica de la adscripción institucional se pueden interpretar como un cinturón protector del núcleo central del funcionalismo, al igual que los estudios basados en la hipótesis de la selección eran un cinturón protector del enfoque del capital humano. Esto es, son un intento de superar el conjunto de anomalías detectadas reiteradamente dando cabida a otro tipo de variables.

En suma, la investigación que se ha ocupado del funcionamiento del mercado de trabajo académico se puede dividir en dos grandes grupos: los enfoques de carácter interno, que tienen la virtud de observar ciertos procesos de estructuración social en detalle, y los enfoques de carácter externo, que tienen la virtud de observar cómo la ciencia responde a procesos económicos. No obstante, además de los presupuestos teóricos que se muestran inadecuados para el caso de la ciencia, ambos presentan la limitación de no traspasar la frontera que habitualmente les emplaza en las tradiciones disciplinarias y los programas de investigación

\footnotetext{
${ }^{18}$ Además del problema de las distorsiones que provoca la institucionalización del recuento de las citaciones, con los conocidos 'clubes de citas', o las dificultades de que revistas periféricas en términos culturales se incluyan en las bases de datos de prestigio, ahora se está produciendo un cambio del sistema de evaluación desde el 'nivel de impacto' al 'nivel de visibilidad', al considerarse aquél muy limitado. Una revisión crítica de la utilización de la productividad científica puede verse en Fox (1983).
} 
RIS

REVISTA INTERNACIONAL DE SOCIOLOCIAA

No 32, Mayo-Agosto, 2002

en los que surgen y con los que interactúan en el proceso de producción de conocimientos de sus respectivos problemas de estudio. Aún está por desarrollar un modelo teórico que posibilite observar extensamente estos procesos sociales en la ciencia al margen de la productividad y el reconocimiento ${ }^{19}$. A modo de síntesis, los elementos fundamentales que se suelen obviar son las estrategias de selección de empleo de los propios científicos y las estrategias de contratación de las instituciones, lo cuál puede estar basado en los supuestos del intercambio de conocimiento público o en otros supuestos distintos, y las interrelaciones con factores externos, a saber, el factor del mercado a través del número de científicos y de lugares o puestos susceptibles de ocuparse, el papel de las actuaciones políticas en la regulación de las instituciones científicas y las posibles influencias derivadas de la relación de la ciencia con el entorno social.

Algunas de las limitaciones señaladas suelen provenir de la interpretación que del mundo de la ciencia se solía hacer con anterioridad a los años 1980, que emplazaba a la ciencia académica en una especie de torre de marfil, con sus propias reglas de funcionamiento y estructuras organizativas, en todo caso muy distintas a las de la ciencia industrial. No obstante, a partir de dichos años surgen algunos enfoques relevantes en el ámbito de la sociología y de la política de la ciencia que contribuyen a enriquecer los marcos de análisis desde los que se observa esta institución al hilo de los profundos cambios que se producen en la ciencia contemporánea. Dichos cambios se refieren a la extensión de la ciencia a entornos organizativos muy diversos, tanto públicos como privados, y a la transformación que están experimentando los sistemas nacionales de I+D, en especial las instituciones de la ciencia pública, que cada vez más incorporan mecanismos de gestión y nociones valorativas que se consideraban propios de la ciencia industrial ${ }^{20}$. Las ideas contenidas en la investigación sobre las nuevas formas de organización social de la ciencia son las que proveen un marco general más adecuado para contextualizar el sistema de ocupaciones científicas, tanto en

\footnotetext{
${ }^{19}$ A pesar del radical cambio de paradigma llevado a cabo por las posteriores sociologías del conocimiento científico, la reformulación de los conceptos del funcionalismo en los estudios empíricos sobre desigualdad en la ciencia se abandonó casi definitivamente, así como la investigación empírica en este campo.

${ }^{20}$ Los principales exponentes de estos estudios son, de un lado, el trabajo ' $L a$ nueva producción de conocimientos' (Gibbons et al.,1994), con los conocidos 'modo 1' y 'modo 2', etiquetas que resumen conjuntos de características propias de un estadio de producción de conocimientos, en este caso los de la ciencia académica y los de la ciencia diversificada dentro y fuera de la fronteras disciplinarias. De otro lado, es Ziman el autor que ha planteado un conjunto formalizado opuesto a las normas funcionalistas, con otro acrónimo simbolizado por la palabra PLACE -Propietaria, Local, Autoritaria, Comisionada y Experta- como resumen de la serie de características del nuevo estadio de la ciencia (Ziman, 1989; 1996). Si el acrónimo CUDOS se refería a los valores claves que guiaban la conducta de los científicos - la fama o crédito-la palabra place simboliza la realización de la ciencia en el contexto de aplicación y las fuerzas que influyen en ese contexto. Ambos modelos
} 


\begin{tabular}{rr} 
R IS \\
MERCADOS DE TRABAJO EN LA CIENCIA \\
\hline
\end{tabular}

la ciencia académica como industrial, y para observar las especificidades de los mercados de trabajo en los distintos entornos.

\section{LA DEFINICIÓN DE LOS ENTORNOS DE INVESTIGACIÓN}

Una vez revisadas las principales aportaciones que tienen que ver con la estructura ocupacional de la ciencia, el siguiente objetivo es establecer un marco analítico que posibilite la observación del funcionamiento de los mercados de trabajo en los distintos entornos teniendo en cuenta la diversidad de organizaciones científicas y las distintas lógicas y modos de actuación que ocurren en ellas. Por ese motivo se utiliza el plural, se habla de mercados de trabajo, no de mercado en general, debido a que la realidad muestra tipos de intercambio que, si bien son comunes en lo referido al objeto de trabajo, difieren tanto en los criterios de selección como en las recompensas o incentivos que se utilizan. La estrategia a seguir parte de una descripción general de los mercados de trabajo en la ciencia, para después realizar una operación analítica que identifique las lógicas del intercambio en las instituciones científicas.

\section{El sistema de mercados de la ciencia}

Las instituciones científicas son un caso nítido de mercados de trabajo internos donde existe una lógica de funcionamiento distinta entre los trabajadores de fuera y de dentro. Siguiendo el concepto de mercado interno de Doeringer y Piore, la investigación científica se puede considerar un mercado interno en sus dos versiones. Por una parte, existen diversos mercados ocupacionales para investigadores que disponen de especialización y credenciales concretas. Por otra, este tipo de cualificaciones se circunscriben en numerosos mercados internos en las diferentes instituciones ${ }^{21}$. La evidencia de las distintas investigaciones indica que

responden a conjuntos característicos de prácticas sociales observables en la vida científica contemporánea y hacen referencia a la variedad de tareas que se espera que hagan los científicos a lo largo de sus carreras profesionales en función del tipo de institución en que trabajan. Por otra parte, estudios sobre los cambios existentes tanto en la ciencia académica como en la universidad pueden verse en Cozzens et al. (1989); Zimberg (1991) y Wasser (1990).

${ }^{21}$ Uno de los puertos de entrada a los mercados de trabajo internos son las becas de investigación o los research assistants, que se conforman habitualmente como un punto de paso obligatorio y que, a la vez, funcionan como situaciones de espera en la cola de acceso a las ocupaciones más estables y mejor remuneradas. El otro puerto para el caso de las universidades son los puestos de profesor ayudante o de profesor asociado, ya que esta ocupación se utiliza habitualmente como elemento de formación y de espera para el acceso a puestos de mayor estatus. 


\section{RIS}

REVISTA INTERNACIONAL DE SOCIOLOCIA

No 32, Mayo-Agosto, 2002

MANUEL FERNÁNDEZ ESQUINAS

los mercados de trabajo en la ciencia son efectivamente internos y relativamente estancos, en el sentido de que existen mecanismos que favorecen el reclutamiento de trabajadores situados en la cola de una institución o frenan el reclutamiento de trabajadores con procedencias distintas. Las instituciones académicas tienden a reclutar a sus trabajadores en las sucesivas posiciones de la estructura ocupacional entre las personas que constituyen la cantera y que ya han accedido al mercado interno por una de las vías establecidas. De esta forma, las fuerzas del mercado, como la oferta de trabajadores en una especialidad concreta y su competencia basada en el currículum científico, están frecuentemente al margen del funcionamiento del mercado interno de una institución.

Figura 1.

Sistema de mercados en la Ciencia *

\begin{tabular}{|l|c|c|c|c|c|}
\hline PRODUCTO & COMPRADOR & VENDEDOR & $\begin{array}{c}\text { MEDIO } \\
\text { PRINCIPAL DE } \\
\text { PAGO }\end{array}$ & $\begin{array}{c}\text { INSTITUCIÓN } \\
\text { TIPO }\end{array}$ \\
\hline $\begin{array}{l}\text { Mercado } \\
\text { reputacional }\end{array}$ & $\begin{array}{c}\text { Artículos } \\
\text { Tesis }\end{array}$ & $\begin{array}{c}\text { "Colegios } \\
\text { invisibles" }\end{array}$ & $\begin{array}{c}\text { Investigadores } \\
\text { Grupos } \\
\text { Instituciones }\end{array}$ & Reconocimiento & $\begin{array}{c}\text { Comunidad } \\
\text { cientíica }\end{array}$ \\
\hline $\begin{array}{l}\text { Mercado de } \\
\text { investigación } \\
\text { privado }\end{array}$ & $\begin{array}{c}\text { Informes } \\
\text { Patentes }\end{array}$ & $\begin{array}{c}\text { Instituciones } \\
\text { Públicas }\end{array}$ & $\begin{array}{c}\text { Grupos } \\
\text { Instituciones }\end{array}$ & Dinero/recursos & $\begin{array}{c}\text { Unidad de I+D } \\
\text { en la empresa }\end{array}$ \\
\hline $\begin{array}{l}\text { Mercado de } \\
\text { investigación } \\
\text { público }\end{array}$ & $\begin{array}{c}\text { Conocimiento } \\
\text { público }\end{array}$ & $\begin{array}{c}\text { Agencias } \\
\text { investigación } \\
\text { Agencias sin }\end{array}$ & $\begin{array}{c}\text { Investigadores } \\
\text { Instituciones }\end{array}$ & $\begin{array}{l}\text { Dinero/recursos } \\
\text { Reconocimiento }\end{array}$ & $\begin{array}{c}\text { Centro de } \\
\text { investigación } \\
\text { público }\end{array}$ \\
\hline $\begin{array}{l}\text { Mercado } \\
\text { educacional }\end{array}$ & $\begin{array}{c}\text { Educación } \\
\text { superior } \\
\text { Títulos }\end{array}$ & Estudiantes & Instituciones & Dinero/recursos & $\begin{array}{c}\text { Universidad } \\
\text { orientada a } \\
\text { docencia }\end{array}$ \\
\hline $\begin{array}{l}\text { Mercado de } \\
\text { trabajo }\end{array}$ & $\begin{array}{c}\text { Fuerza de } \\
\text { trabajo }\end{array}$ & Instituciones & Investigadores & $\begin{array}{l}\text { Dinero/recursos } \\
\text { Reconocimiento }\end{array}$ & \\
\hline
\end{tabular}

* Un primer esbozo de este esquema, aplicado al reclutamiento de los científicos formados en España, se incluye en Fernández Esquinas (2002) 
A pesar de la utilidad de la perspectiva institucional, hay que añadir que el concepto "mercado interno" presenta limitaciones debido a que los modos de operar pueden responder a distintos contenidos del trabajo de los científicos. Así, un recurso útil para avanzar en el entendimiento de la complejidad de este entramado respecto a la selección de sus trabajadores es mediante las formas en que las instituciones interactúan con su entorno. Y para ello se puede acudir a la idea del mercado como metáfora que permite delimitar las diferentes relaciones de intercambio de las instituciones científicas. Esta idea ofrece algunas claves para entender la lógica de estas relaciones, al mismo tiempo que provee de un marco analítico para diferenciar distintos tipos de mercado de trabajo respecto a su funcionamiento interno, teniendo en cuenta que no todas las instituciones científicas presentan la misma realidad respecto a sus actividades ${ }^{22}$. El concepto de mercado se toma en uno de sus significados más simples. Un mercado es un lugar para el intercambio sistemático de productos por medios de pago entre compradores y vendedores. Aunque el símil no parezca tener mucha utilidad económica, sí tiene utilidad sociológica debido a que permite distinguir entre los distintos actores y entre las distintas lógicas en las que se basa el intercambio. Por tanto, el mercado no se considera en su sentido económico, sino que incluye las convenciones sociales, los límites institucionales, las regulaciones organizativas y los contextos políticos en los que se enmarca. En concreto, en las instituciones de la ciencia en términos analíticos se pueden delimitar varios tipos de relaciones de intercambio superpuestas que pueden ser entendidas a partir de los elementos básicos que existen en un mercado: productos, compradores, vendedores y medios de pago.

El 'mercado reputacional' constituye la concepción clásica de la ciencia desarrollada principalmente por la corriente funcionalista. El principal producto que se intercambia es el conocimiento sancionado, que adopta la forma de documentos públicos puestos a disposición de la comunidad científica. De este modo, siguiendo con la metáfora, los bienes que se canjean son artículos en revistas científicas, tesis doctorales y ciertos tipos de libros. Los interesados en producir e intercambiar estos productos son los propios investigadores, como medio de recibir una de las recompensas de la ciencia, como es el reconocimiento por las contribuciones realizadas. No obstante, no sólo son los individuos los interesados en este tipo de intercambio. El crédito trasciende los nombres de los investigadores y es utilizado por las instituciones como elemento que provee de

\footnotetext{
${ }^{22}$ La idea se ha tomado de un artículo del profesor Ziman (1991). Por otra parte, la terminología del mercado ha sido utilizada frecuentemente en los estudios sociales de la ciencia como recurso para resaltar la dimensión utilitaria en los comportamientos de los científicos. Ver, por ejemplo, Bourdieu (1988) y Latour y Woolgar (1979).
} 
RIS

prestigio, pudiéndose extender a ámbitos nacionales ${ }^{23}$. Como compradores en este intercambio se pueden considerar a los miembros de la comunidad científica de cada especialidad, comunidad formada por los paneles de evaluadores de las revistas, que sancionan las contribuciones relevantes al conocimiento mediante el acceso a los medios de comunicación, y por la audiencia de la disciplina que otorga prestigio formal mediante las citas o informal a través de la reputación profesional.

El segundo mercado es el 'mercado de investigación público', y refleja la relación de los investigadores e instituciones con el sistema de financiación estatal, normalmente a través de las agencias especializadas que distribuyen fondos públicos, pero también de las agencias supranacionales o de agencias privadas sin ánimo de lucro. Ciertamente, puede resultar algo confuso pensar en este hecho a modo de mercado, pero tanto los productos como los actores se pueden adaptar fácilmente a este esquema en tanto que se produce una relación de intercambio. Los productos son conocimientos, pero aquí se ha preferido separarlos de las publicaciones y resaltar su posible uso. Los compradores son los propios estados utilizando como intermediarios a grupos de científicos, por ejemplo, mediante el sistema de evaluación por pares. Los estados son los que demandan actividades de investigación a través de la financiación de proyectos dirigidos a objetivos considerados de interés general. Sin embargo, no son los últimos consumidores, sino que financian proyectos para que sus resultados sean de dominio público. Es por ello por lo que se utiliza el calificativo 'público', porque el objetivo es producir conocimiento no privatizable. Este tipo de producto es intercambiado por investigadores e instituciones como forma de lograr uno de sus objetivos: las contribuciones al desarrollo de la ciencia. Es decir, es la forma de entrar en el mercado anterior. Los medios de pago son obviamente recursos para investigación, sea en forma de dinero o infraestructuras, aunque dado que esta financiación es competitiva también se utiliza como un factor de prestigio o reconocimiento.

El tercero de los mercados es el 'mercado privado de los productos de investigación'. Los bienes que se intercambian son patentes, asesorías técnicas o informes, y constituyen un conocimiento apropiable de forma exclusiva por un determinado cliente. Si los resultados de la investigación se hacen públicos es después de ser rentabilizados. El perfil del comprador es el que desea la utilización directa del conocimiento producido, ya sea a través de la compra de un informe $o$ de la explotación de una patente. En este mercado el interés de los vendedores es distinto debido a que la publicidad de los resultados se pospone a otro intercambio

\footnotetext{
${ }^{23}$ Por ejemplo, la producción científica en bases de datos internacionales a través del número de artículos o su índice de impacto son algunos de los indicadores habitualmente utilizados para medir la capacidad científica de un pais.
} 
de tipo monetario. Esto responde al deseo de obtener ingresos por parte de los investigadores, pero también a la necesidad de obtener fondos alternativos que complementen una financiación pública insuficiente para mantener los grupos de investigación, a la imposibilidad de entrar en los mercados anteriores debido a la falta de una reputación que permita competir e, incluso, a la búsqueda de legitimación y utilidad de las actividades de una institución pública. Por último, el 'mercado educacional' constituye la relación de intercambio principal de las instituciones académicas, donde el producto son servicios educativos y, en última instancia, títulos de educación superior, canjeados entre los estudiantes que los adquieren y las universidades que los proveen. El medio de pago son los recursos, bien aportados directamente por los estudiantes, bien a través de subvenciones estatales a las universidades realizadas normalmente en función del número de estudiantes.

\section{Tipos ideales e interacción de mercados}

Una de las posibilidades que presenta este esquema es la delimitación de las instituciones científicas en función del mercado predominante en el que actúan. Aunque uno de los señalados es principalmente simbólico -el mercado reputacional que utiliza la comunidad científica - se pueden identificar instituciones típicas en las que predomina alguno de los restantes mercados. Para nuestro propósito, el esquema anterior permite identificar las lógicas de funcionamiento del mercado de trabajo, que se pueden considerar coherentes con el sistema de intercambio dominante o, lo que es lo mismo, que son coherentes con la orientación de la institución respecto a los intercambios con su entorno. La selección de los recursos humanos en una organización científica depende de la orientación de sus unidades a uno o varios de los mercados, así como de la estrategia empleada por el gobierno de la organización o por sus diversas unidades. De esta forma, los criterios seguidos en la selección pueden adoptar varias estrategias que permiten afianzar su posición en la relación de intercambio. Siguiendo con el esquema, existen varios escenarios respecto al funcionamiento interno del mercado de trabajo tanto desde el punto de vista del gobierno de las instituciones como de sus unidades de investigación.

El primer caso es el de los grupos orientados a la producción de conocimientos que sean sancionados positivamente por la comunidad de expertos. El estatus del grupo se basa en la competencia en el sistema de recompensas de la ciencia a través de las publicaciones, mediante las cuales se accede al ciclo de credibilidad que permite obtener fondos para realizar investigación. En este contexto es crucial para el grupo disponer de trabajadores que contribuyan al aumento de la credibilidad, por lo cual la estrategia de formación y selección de personal está dirigida al reclutamiento de trabajadores que contribuyan a la producción científica. Este es el caso del mercado de trabajo basado en una competición de 
carácter reputacional, en el que las distintas instituciones luchan por atraer a los profesores e investigadores más prestigiosos y con más posibilidades de producción científica. El currículum científico centrado en las publicaciones aparece aquí como el principal determinante de la selección, y es donde el mecanismo de la meritocracia basado en las contribuciones a la ciencia está más presente. Un segundo caso es el de grupos o departamentos orientados a las áreas más aplicadas. En este caso, la supervivencia del grupo depende del intercambio de productos con el exterior, en función de la utilidad que éstos tengan para un determinado cliente. En la selección de personal, los logros publicados son relevantes, pero quizá no son lo más importante para la estrategia del grupo. Es decir, el ciclo de credibilidad es distinto, no está basado en el reconocimiento formal de la comunidad científica, sino en un grupo reducido de usuarios. De esta forma, en la selección de trabajadores cobra mayor importancia la disposición de un conjunto de cualidades estratégicas para el trabajo de investigación, estén o no refrendadas por el reconocimiento público. El tercer caso es el de los grupos orientados hacia la docencia, en los que la investigación no es parte fundamental del trabajo, o bien se realiza con carácter individual. Los criterios de reclutamiento pueden ser una combinación de currículum docente y científico, pero en este caso ni el prestigio ni la disposición de cualidades específicas para la investigación son fundamentales, en tanto que los intercambios se basan en servicios proporcionados a los estudiantes. La investigación tiende a entenderse más bien como factor de prestigio o como complemento beneficioso para la docencia.

El esquema también proporciona posibilidades para analizar la interacción entre los distintos componentes, sobre todo en los vínculos con el mercado reputacional. La relación entre el mercado constituido por la financiación pública competitiva está en estrecha relación con la formación del ciclo de credibilidad de los científicos sobre la base de la reputación (Latour y Woolgar, 1979). La realización de contribuciones al conocimiento en el sector industrial es publicada tras su explotación comercial, como forma de incrementar el prestigio y la publicidad de la empresa, interactuando del mismo modo con el mercado reputacional. Así mismo, para los investigadores individuales, dada la conexión de la investigación aplicada con importantes avances del conocimiento, la investigación privada les permite conseguir ciertas recompensas simbólicas que son compatibles con las pautas que frenan la publicidad. Por ejemplo, además de los artículos publicados, en ciertas disciplinas se considera relevante acompañar un currículum de investigación con algunas patentes y asesorías técnicas. Por último, la relación del mercado educacional con el mercado reputacional se puede ejemplificar en las universidades de sistemas competitivos, en las que se utiliza a científicos reconocidos para conseguir mayor prestigio, para incrementar los precios de las matrículas y para obtener una mayor demanda por parte de los estudiantes, primando para ello con mayores salarios la productividad de los científicos que contratan (Gómez Mejía y Balkin, 1992). 
Los tres casos mencionados se pueden considerar como tipos ideales de contextos de investigación en los que existen prácticas de selección de personal coherentes con los distintos tipos de interacción con el entorno, a saber: el mercado basado en la reputación, el mercado de trabajo de científicos aplicados y el mercado docente, y pueden aportar un marco útil para identificar modos de funcionamiento del mercado de trabajo. Aunque aún no se dispone de suficiente evidencia para delimitar empíricamente un tipo u otro de orientación, parece un hecho constatado que las instituciones en las que existe un mayor contenido docente del trabajo es en las universidades y, dentro de ellas, en las áreas menos experimentales. Por tanto, se puede plantear como hipótesis que en el modelo de selección de trabajadores está menos presente la reputación y las cualificaciones concretas, simplemente porque en estas instituciones existen menos intercambios con el entorno en términos de producción científica. Desde luego, cualquier gobierno de una universidad o centro público prefiere tener a científicos de prestigio entre sus profesores. Pero en las universidades en las que ni la financiación pública, ni el número de estudiantes, ni los ingresos que reciben por las tasas dependen del prestigio científico, la disposición de un perfil de trabajador definido no es un requisito fundamental para el mantenimiento o la expansión de la institución. Por otra parte, la orientación al mercado reputacional tiende a ser más utilizada por grupos de científicos consolidados, que basan su prestigio en la realización de avances al conocimiento público, en los que se busca un perfil profesional acorde con el grupo de iguales.

El esquema anterior también proporciona un marco que contribuye a mostrar la diversidad de opciones y posibilidades que ocurren en las instituciones científicas así como a invalidar los argumentos simples que se suelen emplear respecto al reclutamiento de científicos académicos, aclarando la complejidad de los procesos sociales que se ocultan tras este fenómeno. En los grupos basados en el intercambio de recursos a cambio de credibilidad se suelen necesitar trabajadores que, además de disponer de un currículum adecuado, dispongan de destrezas concretas, como por ejemplo el manejo de equipos altamente especializados o el conocimiento en profundidad de los procesos de trabajo de un lugar concreto. Esto es, en la investigación científica, además del conocimiento formalizado, siempre existe un conocimiento tácito que es difícil de evaluar a través de referencias externas al proceso de trabajo. Del mismo modo, en la investigación aplicada, ante la incertidumbre de obtener resultados útiles, los consumidores de productos de investigación suelen utilizar como referente el prestigio de los científicos. Por último, no hay que olvidar que existen instituciones académicas que interactúan con los tres tipos de entornos fruto de la diversificación de actividades que se observan en las universidades y los centros públicos de investigación. 
RIS

\section{HACIA UNA DEFINICIÓN OPERATIVA PARA LA INVESTIGACIÓN DEL MERCADO DE TRABAJO CIENTÍFICO}

A partir de la anterior definición literaria, es posible construir una formulación en términos operativos que delimite las principales dimensiones y variables a tener en cuenta en la investigación empírica. Previamente, se especifican las unidades de análisis sobre las que se centra la observación, que constituyen las grandes dimensiones en función de qué unidad de análisis se considere prioritaria. En concreto, se establecen cuatro niveles, correspondientes a la organización, la unidad organizativa, el puesto de trabajo y el trabajador o candidato. Hay que decir que, obviamente, no se deben confundir las dimensiones con las unidades de análisis. Esto es, es posible establecer como unidad de análisis una de las cuatro citadas, identificando dimensiones de dicha unidad que se atribuyen a ella misma y a las tres restantes, o bien establecer varias unidades de análisis, aunque esta operación puede presentar limitaciones para análisis de datos conjuntos en los que se quieran observar interacciones de variables presentes en varias dimensiones. Por otra parte, si bien la organización es la que enmarca socialmente el proceso de trabajo y el empleo de los científicos, suele ser la unidad organizativa la instancia que realiza la tarea científica debido a la alta autonomía que tiene la investigación teniendo en cuenta la complejidad y la necesidad de especialización. Así, los controles se realizan en las propias unidades organizativas, quedando para las organizaciones los controles externos al proceso concreto de trabajo. Además, también hay que diferenciar entre las características de los puestos, que son las que definen las posibilidades de trabajo, y las características de los trabajadores, tanto los aspirantes como los finalmente elegidos.

Respecto a cada una de las unidades, la primera operación consiste en aislar los elementos que son susceptibles de observación a modo de subdimensiones, así como establecer un esbozo de las posibles mediciones a través de variables (ver figura 2). Cabe mencionar que un lugar importante lo ocupan las características del entorno con el que interactúa una organización o una unidad organizativa, debiéndose definir cada uno de los sistemas de intercambio especificados antes, a saber: el intercambio de recursos con las instituciones financiadoras de la ciencia pública, basado o no en la contribución al conocimiento público, el intercambio de aplicaciones privadas o el de servicios educativos. Así, cobran relevancia el tipo de actividad principal que desarrolla la institución y las características y organización de los puestos de trabajo que incorpora. En cuanto a las dimensiones correspondientes a los puestos y los trabajadores científicos, las principales corresponden a las características de su formación, a la trayectoria ocupacional previa, incluyendo los logros científicos, y a la interiorización de uno de los sistemas de recompensas de la ciencia, o lo que es lo mismo, el sistema de valores en torno a los intercambios que se producen respecto a los productos de investigación del trabajador científico. La definición operativa que aquí se propone pretende tener 
RIS

REVISTA INTERNACIONAL DE SOCIOLOCI

№ 32, Mayo-Agosto, 2002

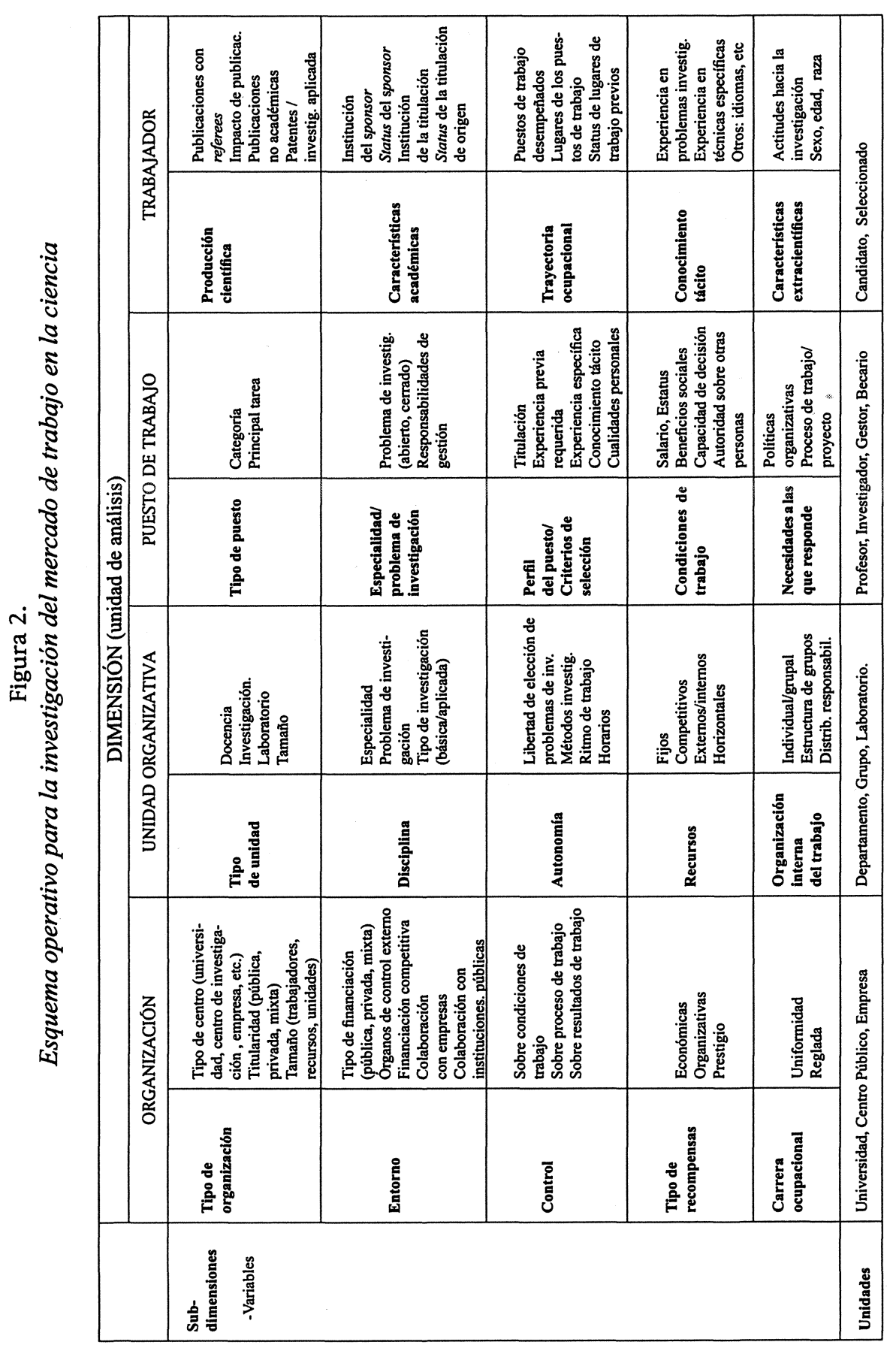

69 
en cuenta simultáneamente cada una de las dimensiones y subdimensiones, si bien la principal unidad de análisis que se establezca, a la que se pueden subordinar las restantes en la estrategia de recogida de datos y análisis, dependerá del objetivo principal de cada investigación, que responderá a su vez al planteamiento de problemas de estudio concretos en función del programa de investigación del mercado de trabajo que se adopte.

\section{CONCLUSIONES}

Las ideas aquí recogidas muestran que la ciencia no está aislada en una especie de torre de marfil y que las condiciones sociales de su entorno funcionan a la vez como condiciones sociales de la ciencia, lo que plantea el requerimiento de utilizar un marco de análisis no reduccionista en la investigación de cualquier parcela de la vertiente social de la ciencia, como es el funcionamiento de sus mercados de trabajo. La propuesta que se presenta será o no relevante en función de la contrastación empírica de sus presupuestos, quedando pues por demostrar la validez de su diseño, aunque al menos en términos analíticos queda también por responder una de las cuestiones que se señalaban como limitadores de los estudios existentes, la dicotomía interno-externo. Se es consciente de que aquí se ha realizado una operación referida principalmente a la lógica específica que opera en el interior de las instituciones científicas al ser ésta muy diferente a la de otras instituciones. Ello ha sido así porque se asumía que la principal carencia de los Estudios Sociales de la Ciencia estaba en definir un marco analítico que tuviera en cuenta la compleja realidad huyendo en lo posible de ideas preconcebidas. Si bien la operacionalización del entorno da idea de las influencias de éste en la configuración del mercado de trabajo, es necesario especificar también las características de dicho entorno y delimitar cómo interviene en la creación de puestos de trabajo de carácter científico, en la forma en que se ocupan los puestos y en las consecuencias que dichos condicionantes tienen en la relevancia científica, social y económica de los sistemas de I+D. Aunque también se encuentran notables ejemplos en la literatura, es aún necesario analizar y operacionalizar la influencia de cuestiones externas en el funcionamiento y los resultados de los mercados de trabajo, a saber, las influencias del propio mercado en términos de oferta y demanda, tanto local como internacional, las influencias de la actuación del Estado y de los organismos internacionales, las que provienen del desarrollo de la investigación científica en sus principales disciplinas y de la situación organizativa de los sistemas de investigación en los que se encuadran las instituciones y los trabajadores científicos. 


\section{REFERENCIAS BIBLIOGRÁFICAS}

ALBURY, B. y J. SCHWARD (1982), Partial Progress. The Politics of Science and Technology, Londres, Pluto Press.

ALLEN,T.J. y R. KATZ (1986), “The Dual Ladder: Motivational Solutions or Managerial Delution?”, $R \& D$ Management, $\mathrm{n}^{\circ} 16, \mathrm{pp} .185-197$.

BECKER, G. (1983), El capital humano, Madrid, Alizanza Editorial.

BECKER, H.S. y J. CARPER (1956), "The Elements of Identification with an Occupation", American Sociological Review, vol. 21, pp. 341-348.

BEN-DAVID, J. (1972), "The Professions of Science and its Powers", Minerva, vol.10, pp. 362-383.

BERG, I. (ed.) (1981), Sociological Perspectives on Labor Markets, Nueva York, Academic Press.

BLAUG, M. (1983), "El estatus empírico de la teoría del capital humano", en L. Toharia (ed.), $E l$ mercado de Trabajo. Teorias y aplicaciones, Madrid, Alianza Editorial

BRENEMAN, D.V. y T.I.K. YOUN (eds.) (1988), Academic Labor Market and Careers, Philadelphia, The Falmer Press.

BOURDIEU, P. (1988), Homo Academicus, Cambrigde, Polity Press.

BURKE, D.L. (1988), A New Academic Marketplace, Nueva York, Grenwood Press.

CAPLOW, Th. y R.J. MCGEE (1958), The Academic Marketplace, Nueva York, Science Editors Inc.

(1968), “Procedures of Academic Recruitment”, en B.G. Glaser (ed.), Organizational Careers: A Source Book for Theory, Chicago, Aldine Publishing Co.

CARTTER, A.M. (1965), "The Demand and Supply of College Teachers", Social Statistic Proceedings, pp. 70-80.

CLARK, B.R. (ed.) (1987), The Academic Profession, Berkeley, UCA Press.

COLE, S. y J.R. COLE (1967), "Scientific Output and Recognition: a Study in the Operation of the Reward System in Science”, American Sociological Review, $\mathrm{n}^{\circ} 32$, pp. 377-390.

(1973), Social Stratification in Science, Chicago, University of Chicago Press.

COLLINS, R. (1981), “Crises and Declines of Credential Systems”, en VV.AA., Sociology Since Mid-century, Nueva York, Academic Press.

CORTGROVE, S. y S. BOX (1970), Science, Industry and Society. Studies in the Sociology of Science, Londres, George Allen and Unwin. 


\section{RIS}

COZZENS, S.E., P. HEALEY, A. RIP y J.ZIMAN (eds.) (1989), The Research System in Transition, Dordrech, Kluwer Academic Publishers.

CRANE, D. (1965), "Scientist at Major and Minor Universities: a Study of Productivity and Recognition", American Sociological Review, $\mathrm{n}^{\circ}$ 30, pp. 700-715.

(1969), "Social Structure in a Group of Scientists: a Test of the 'Invisible College' hypothesis", American Sociological Review, vol. 34, pp. 335-352.

(1971), "The Academic Market Place Revisited: a Study of Faculty Mobility Using the Cartter Ratings", American Journal of Sociology, $\mathrm{n}^{\circ}$ 7, pp. 953-964.

DEBACKERE, K. y M.A. RAPPA (1995), "Scientist at Major and Minor Universities: Mobility along the Prestige Continuum", Research Policy, n² 24, pp. 137-150.

DOERINGER, P.B. y M.J. PIORE (1971), Internal Labour Markets and Manpower Analisys, Lexinton, Heath Lexinton Books.

EIDUSON, B.T. y L. BECKMAN (eds.) (1973), Science as a Career Choice. Theoretical and Empirical Studies, Nueva York, Russell Sage Foundation.

FERNÁNDEZ ESQUINAS, M. (2002), La formación de investigadores cientificos en España, Madrid, CIS.

FREEMAN, R. (1971), The Market for College-tained Manpower. A Study in the Ecocomics of Careers Choice, Cambrigde, Harvard University Press.

FREEMAN, R.B. (1975), "Supply and Salary Adjustement to the Changing Science Manpower: Phisics 1948-73", American Economic Review, 65, pp. 27-39.

(1994), Mercados de trabajo en acción. Ensayos sobre economía empirica, Madrid, Ministerio de Trabajo y Seguridad Social, Colección economía y sociología del Trabajo, $\mathrm{n}^{\circ} 72$.

FOX, M.F. (1983), "Publication Productivity Among Scientist: a Critical Review", Social Studies of Science, vol.13, pp. 285-305.

FRIEDKIN, N. E. (1978), "University Social Structure and Social Network among Scientists", American Journal of Sociology, vol. 83, n.6, pp. 1444-1465.

GASTON, J. (1978), The Reward System in British and American Science, Nueva York, Wiley and Sons.

GIBBONS, M., C. LIMOGES, H. NOWOTNY, S. SCHWARTZMAN, P. SCOTT y M. TROW (1994), The New Production of Knowlegde. The Dynamics of Science and Research in Contemporary Societies, Londres, Sage.

GILBERG, G.N. (1977), "Competition, Differentiation and Careers in Science", Social Science Information, vol. 16, pp. 103-123. 
GLASER, B.G. (1967), Organizational Scientists: their Professional Careers, Indianapolis, The Bobs-Merrill Company, Inc.

GÓMEZ MEJ́A, L. y D.B. BALKIN (1992), "Determinants on Faculty Pay: an Agency Theory Perspective", Academy of Management Journal, vol. 35, pp. 921-955

GÓMEZ MEJÍA, L. y M.W. LAWLESS (eds.) (1992), Global High-Technology Management, Greenwich-London, JAI Press.

GORDON, M. (ed.) (1974), Higher Education and the Labor Market, Nueva York, McGraw-Hill.

HANGSTROM, W.O. (1965), The Scientific Community, Nueva York, Basics Books.

HARGENS, L.L. y W.O. HANGSTROM (1967), "Sponsored and Context Mobility of American Academic Scientists", Sociology of Education, vol.10, $\mathrm{n}^{\circ} 1, \mathrm{pp} .25-38$.

KALLENBERG, A.L. y A.B. SORENSEN (1979), "The Sociology of Labor Market", Annual Review of Sociology, vol.5.

KORNHAUSER, W. (1962), Scientists in Industry: Conflict and Accommodation, Berkeley, UCA Press.

KROHN, R.G. (1971), The Social Shaping of Science. Institutions, Ideology and Careers in Science, Westport, Greenwood Pub.

LATOUR, B. y S. WOOLGAR (1979), Vida de laboratorio, Madrid, Alianza Editorial.

LONG, J. S., P.D. ALLISON y R. MCGINNIS (1979), "Entrance into the Academic Career", American Sociological Review, vol. 44, pp. 816-830.

LONG, J. S. y R. MCGINNIS (1981), “Organizational Context and Scientific Productivity”, American Sociological Review, vol. 46, pp. 422-442.

LONG, J. S. y M.S. FOX (1995), "Scientific Careers: Universalism and Particularism", Annual Review of Sociology, $\mathrm{n}^{\circ}$ 21, pp. 45-71.

MERTON, R. K. (1977), Sociología de la ciencia, vol. II. Madrid, Alianza Editorial.

(1977a), "La estructura normativa de la ciencia", en R.K. Merton op.cit. (1977).

MERTON, R.K. y H. ZUCKERMAN (1977), "Pautas institucionalizadas de evaluación en la ciencia", en R.K. Merton op.cit., (1977).

MARCSON, S. (1968), “Career Development of Scientists”, en B.G. Glaser (ed.), Organizational Careers: a Source Book for Theory, Chicago, Aldine Publishing Co.

MILLER, H. (1996), “Academics and Their Labour Process”, en C. Smith., D. Knights y H. Willmott (eds.), White-collar Work. The Non-manual Labour Process, Londres, Macmillan. 
RIS

REVISTA INTERNACIONAL DE SOCIOLOGIA

N 32, Mayo-Agosto, 2002

MANUEL FERNÁNDEZ ESQUINAS

MINCER, J. (1971), Schooling, Age and Earnings, Nueva York, National Bureau of Economic Research.

MULKAY, M. (1976), "Norms and Ideology in Science", Social Science Information, $\mathrm{n}^{\circ} 15, \mathrm{pp}$. 637-656.

NEYMAN, E. (1977), "Scientific Career, Stientific Generation, Scientific Labour Market”, en Blume, S. (ed.), Toward a Political Sociology of Science, Nueva York, Collier-McMillan..

NILAND, J.R. (1971), “Allocation of Phd Manpower in the Academic Labor Market”, Industrial Relations, $\mathrm{n}^{\circ} 2$, pp. 141-156.

PIORE, M.J. (1975), "Notas para una teoría de la estratificación del Mercado de Trabajo", en Toharia, L. (ed.), El mercado de trabajo. Teorias y aplicaciones, Madrid, Alianza Editorial.

POLANYI, M. (1962), "The Republic of Science”, Minerva, vol.1, pp. 54-73.

ROSENFELD, R.A. y J.A. JONES (1987), "Institucional Mobility among Academics", Sociology of Education, $\mathrm{n}^{\circ} 59$, pp. 212-226.

RESKIN, B.F. (1979), “Academic Sponsorships and Scientists' Careers”, Sociology of Education, vol. 52, pp.129-146.

RIESMAN, D. (1968), "Recruitmen to the Academic Career", en Glaser, B.G. (ed.), Organizational Careers: a Source Book for Theory, Chicago, Aldine Publishing Co.

SCOTT, C.D. (1979), “The Market for PhD Economist: The Academic Sector”, American Economic Review, 69, pp. 137-141.

SHENHAV, Y.A. y Y. HABERFELD (1988), "Scientists in Organizations: Discriminations Processes in an Internal Labour Market", The Sociological Quaterly, vol. 29, n 3, pp. 451-462.

SMITH, B.L. y J. KARLESKI (eds.), The State of Academic Science, Nueva York, Change Press.

SMITH, J.P. y F. WELCH (1978), “Overeducated American?”, Proceedings of The National Academy of Education, vol. 5, pp. 49-83.

STIGLER, G. y D. BLANK (1957), The Demand and Supply of Scientific Personnel, Nueva York, NBER.

STIGLITZ, J.R. (1975), "The Theory of the Screening. Education and the Distribution of Income", American Economic Review, $\mathrm{n}^{\circ}$ 65, pp. 315-342.

THUROW, L.C. (1975), Genering Inequality, Nueva York, Basic Books.

TORRES, C. (1995), Sociologia politica de la ciencia, Madrid, CIS.

VENCE, X. (1992), "Tendencias y contradicciones en las relaciones entre ciencia e industria: el proceso de producción científica", en M. Gómez Uranga et al. (comps.), El cambio tecnológico hacia el nuevo milenio. Debates y nuevas teorias, Madrid, FUHEM.

74 
WASSER, H. (1990), "Changes in the European University: from Traditional to Enterpreneurial", Higher Education Quaterly, vol. 44, $\mathrm{n}^{\circ}$ 2, pp. 103-122.

ZIMAN, J. (1989), Researh as a Career, en S.E. Cozzens et al. (eds) (1989).

(1991), “Academic Science as a System of Markets”, Higher Education Quaterly, vol.45, n 1, pp. 41-61.

(1996), "Postacademic Science: Constructing Knowlegde with Networks and Norms", Science Studies, vol. $9, \mathrm{n}^{\circ} 1$.

ZIMBERG, D.S. (1991), The Changing University, Dordrech, NATO ASI Series-Kluwer Academic Publishers.

ZUCKERMAN, H. (1967), "Nobel Laureates in Science: Patterns of Productivity, Collaborations and Autorship", American Sociological Review, vol. 32, n 3, pp. 391-403.

(1970), "Stratification in American science", Sociological Inquiry, $\mathrm{n}^{\circ}$ 40, pp. 235-257.

(1988), "The Sociology of Science", en N. Smelser (ed.), Handbook of Sociology, Londres, Sage.

ZUCKERMAN, H. y J.R. COLE (1975), "Women in American Science", Minerva, no 13, pp. 82 112. 